\title{
NOTES ON INVARIANT SUBSPACES
}

\author{
HARI BERCOVICI
}

\begin{abstract}
The main purpose of this article is to give an approach to the recent invariant subspace theorem of Brown, Chevreau and Pearcy: Every contraction on a Hilbert space, whose spectrum contains the unit circle has nontrivial invariant subspaces. Our proof incorporates several of the recent ideas tying together function theory and operator theory.
\end{abstract}

\section{INTRODUCTION}

The Jordan structure theorem for finite matrices has been known now for over one hundred years, and its usefulness can hardly be overstated. It says that every square matrix $A$ over the complex numbers $\mathrm{C}$ is similar to another matrix $B$ (i.e., $B=X A X^{-1}$ for some invertible matrix $X$ ) which is a direct sum of Jordan cells. That is, $B$ can be written in block form

and each $B_{i}$ has the form

$$
B=\left[\begin{array}{cccc}
B_{1} & 0 & \cdots & 0 \\
0 & B_{2} & \cdots & 0 \\
\cdot & \cdot & \cdots & \cdot \\
0 & 0 & \cdots & B_{k}
\end{array}\right]
$$

$$
B_{i}=\left[\begin{array}{ccccc}
\lambda_{i} & 1 & 0 & \cdots & 0 \\
0 & \lambda_{i} & 1 & \cdots & 0 \\
0 & 0 & \lambda_{i} & \cdots & 0 \\
\cdot & \cdot & \cdot & \cdots & \cdot \\
0 & 0 & 0 & \cdots & \lambda_{i}
\end{array}\right]
$$

for some $\lambda_{i} \in \mathbf{C}$. The numbers $\left\{\lambda_{1}, \lambda_{2}, \ldots, \lambda_{k}\right\}$ can be identified as the spectrum, or set of eigenvalues of $A$,

$$
\begin{aligned}
\left\{\lambda_{1}, \lambda_{2}, \ldots, \lambda_{k}\right\} & =\{\lambda \in \mathbf{C}: \operatorname{det}(\lambda I-A)=0\} \\
& =\{\lambda \in \mathbf{C}: \lambda I-A \text { is not invertible }\} .
\end{aligned}
$$

Received by the editors August 15, 1987 and, in revised form, December 13, 1988.

1980 Mathematics Subject Classification (1985 Revision). Primary 47A15, $47 \mathrm{~A} 45$.

The author wishes to acknowledge the support of the National Science Foundation. 
One can regard $A$ as a linear operator on the finite-dimensional space $\mathbf{C}^{n}$, and then the Jordan theorem says that $\mathbf{C}^{n}$ can be written as a direct sum

$$
\mathbf{C}^{n}=\mathscr{M}_{1} \dot{+} \mathscr{M}_{2} \dot{+} \cdots+\mathscr{M}_{k}
$$

of subspaces such that

(i) each $\mathscr{M}_{i}$ is invariant for $A$, i.e., $A \mathscr{M}_{i} \subset \mathscr{M}_{i}$; and

(ii) the restriction $A \mid \mathscr{M}_{i}$ acts like a Jordan cell.

One particular consequence of this result is that, for $n \geq 2$, $A$ has nontrivial invariant subspaces (nontrivial means different from $\{0\}$ and $\mathbf{C}^{n}$ ).

The search for an analogue of Jordan's theorem for linear operators on infinite-dimensional spaces has led to many interesting developments and, in particular, to an awareness of the fact that in the infinite-dimensional case there is a much greater variety of linear operators. In particular, there are few meaningful questions that one may ask about arbitrary linear operators, with any hope of ever answering them. One such question is the invariant subspace problem which we now formulate. Let $\mathscr{H}$ denote an infinite-dimensional complex Hilbert space, and write $\mathscr{L}(\mathscr{H})$ for the space of all continuous linear operators $T: \mathscr{H} \rightarrow \mathscr{H}$. A subspace of $\mathscr{H}$ is by definition a closed linear manifold in $\mathscr{H}$. A subspace $\mathscr{M} \subset \mathscr{H}$ is nontrivial if $\mathscr{M} \neq\{0\}$ and $\mathscr{M} \neq \mathscr{H} ; \mathscr{M}$ is said to be invariant for $T \in \mathscr{L}(\mathscr{H})$ if $T \mathscr{M} \subset \mathscr{M}$.

1.1. Problem. Does every operator $T \in \mathscr{L}(\mathscr{H})$ have a nontrivial invariant subspace?

One should emphasize the fact that merely knowing the answer to this problem for a given operator $T$ does not give us nearly as much information as the Jordan theorem provides in the finitedimensional case. Nevertheless, the search for nontrivial invariant subspaces usually leads to more information about the given operator.

Many methods have been devised for answering the invariant subspace problem, and we will see several of them in this article. I would like to start by giving some basic examples which show that under certain circumstances one can find more invariant subspaces than expected. For the following discussions we need to recall that an invariant subspace $\mathscr{M}$ for an operator $T$ is said to be reducing for $T$ if the orthogonal complement of $\mathscr{M}$ is also invariant for $T$. Mostly everybody who took a functional 
analysis course must have heard of one of the great success stories in invariant subspace history: the spectral theorem for normal operators (recall that $T$ is normal if $T^{*} T=T T^{*}$ ). The spectral theorem provides a large supply of reducing subspaces such that the normal operator can in fact be constructed if we know these reducing subspaces. Let us focus on a very particular class of normal operators: the diagonal ones. Thus, assume that $\mathscr{H}$ is separable and has an orthonormal basis $\left\{e_{1}, e_{2}, \ldots\right\}$. Given a bounded sequence $w=\left\{w_{1}, w_{2}, \ldots\right\}$ of complex numbers, one can construct an operator $T_{w}$ on $\mathscr{H}$ such that

$$
T_{w} e_{j}=w_{j} e_{j}, \quad j=1,2, \ldots
$$

Such an operator is called a diagonal operator (with diagonal $w$ ). The reducing subspaces of $T_{w}$ are easy to determine if the $w_{j}$ are distinct: given a subset $A$ of $\{1,2,3, \ldots\}$ consider the subspace $\mathscr{M}_{A}$ generated by $\left\{e_{j}: j \in A\right\}$. Then $\mathscr{M}_{A}$ is reducing, and all reducing subspaces of $T_{w}$ have this form (exercise!). Wermer [17] gave an example showing that $T_{w}$ may have nonreducing invariant subspaces, and we would like to consider a closely related example here.

1.2. Theorem. Set $w_{n}=\exp \left(-1 / n^{1 / 2}+i n^{1 / 2}\right)$. Then $T_{w}$ has nonreducing invariant subspaces, but $T_{w}^{-1}$ does not.

Our proof of this theorem will be closer to the approach of Brown, Shields and Zeller [6] than to Wermer's original proof. We need a few preliminary facts. Recall that a subset $A$ of a complex Banach space $\mathscr{X}$ is absolutely convex if $\alpha x+\beta y \in A$ whenever $x, y \in A, \alpha, \beta \in \mathbf{C}$, and $|\alpha|+|\beta| \leq 1$.

1.3. Lemma. Let $\mathscr{X}$ be a Banach space, and let $\left\{x_{n}\right\}_{n=1}^{\infty}$ be a sequence in $\mathscr{X}$ whose closed absolutely convex hull is the unit ball of $\mathscr{X}$. For every $x \in \mathscr{X}$ and every $\varepsilon>0$ there exist scalars $\left\{\alpha_{n}\right\}_{n=1}^{\infty} \subset \mathbf{C}$ such that

$$
\sum_{n=1}^{\infty}\left|\alpha_{n}\right|<\|x\|+\varepsilon \text { and } x=\sum_{n=1}^{\infty} \alpha_{n} x_{n} .
$$

Proof. If $x=0$ we can take $\alpha_{n}=0$ for all $n$. If $x \neq 0$ then $x /\|x\|$ is in the unit ball of $\mathscr{X}$, so there exist scalars $\beta_{n} \in \mathbf{C}$, all zero except finitely many, such that $\sum_{n=1}^{\infty}\left|\beta_{n}\right| \leq 1$, and

$$
\left\|\frac{x}{\|x\|}-\sum_{n=1}^{\infty} \beta_{n} x_{n}\right\|<\frac{\varepsilon}{2\|x\|} .
$$


Setting $\alpha_{n}^{(1)}=\beta_{n}\|x\|$, we have $\sum_{n=1}^{\infty}\left|\alpha_{n}^{(1)}\right| \leq\|x\|$ and

$$
\left\|x-\sum_{n=1}^{\infty} \alpha_{n}^{(1)} x_{n}\right\|<\frac{\varepsilon}{2} \text {. }
$$

Repeating this procedure with $x-\sum_{n=1}^{\infty} \alpha_{n}^{(1)} x_{n}$ in place of $x$, we find $\alpha_{n}^{(2)} \in \mathbf{C}$ such that

$$
\begin{gathered}
\sum_{n=1}^{\infty}\left|\alpha_{n}^{(2)}\right| \leq\left\|x-\sum_{n=1}^{\infty} \alpha_{n}^{(1)} x_{n}\right\|<\frac{\varepsilon}{2}, \text { and } \\
\left\|x-\sum_{n=1}^{\infty} \alpha_{n}^{(1)} x_{n}-\sum_{n=1}^{\infty} \alpha_{n}^{(2)} x_{n}\right\|<\frac{\varepsilon}{4} .
\end{gathered}
$$

This process can be continued inductively to yield $\alpha_{n}^{(k)} \in \mathbf{C}$ such that $\sum_{n=1}^{\infty}\left|\alpha_{n}^{(k)}\right|<\varepsilon / 2^{k-1}$ and

$$
\left\|x-\sum_{j=1}^{k} \sum_{n=1}^{\infty} \alpha_{n}^{(j)} x_{n}\right\|<\frac{\varepsilon}{2^{k}} .
$$

The reader will verify without difficulty that the numbers $\alpha_{n}=$ $\sum_{j=1}^{\infty} \alpha_{n}^{(j)}$ satisfy the requirements of the lemma. Q.E.D.

Let us denote by $\mathbf{D}$ the unit disc in $\mathbf{C}(\mathbf{D}=\{\lambda \in \mathbf{C}:|\lambda|<1\})$, and write $H^{\infty}$ for the Banach algebra of all bounded analytic functions in $\mathbf{D}$ (with the sup norm). A subset $\Lambda \subset D$ is said to be dominating [15] if $\sup \{|u(\lambda)|: \lambda \in \Lambda\}=\|u\|_{\infty}(=\sup \{|u(\lambda)|: \lambda \in$ D\}) for every $u \in H^{\infty}$. Let us recall that every function $u \in H^{\infty}$ has nontangential limits $u(\zeta)$ for almost every $\zeta$ in the unit circle $\mathbf{T}=\{\zeta:|\zeta|=1\}$, and

$$
\|u\|_{\infty}=\operatorname{ess} \sup \{|u(\zeta)|: \zeta \in \mathbf{T}\}
$$

It follows immediately that a set $\Lambda$ is dominating if almost every point in $\mathbf{T}$ is a nontangential limit of $\Lambda$. (The converse is also true; cf. [6].)

1.4. Lemma. The set $\left\{w_{n}\right\}_{n=1}^{\infty}$, where $w_{n}=\exp \left(-1 / n^{1 / 2}+i n^{1 / 2}\right)$, is dominating.

We leave the proof of this lemma as an exercise, but the following picture tells almost all the story.

We will need now a basic fact about the space $H^{\infty}$ : it is (isometrically isomorphic to) the dual of a Banach space. One can 


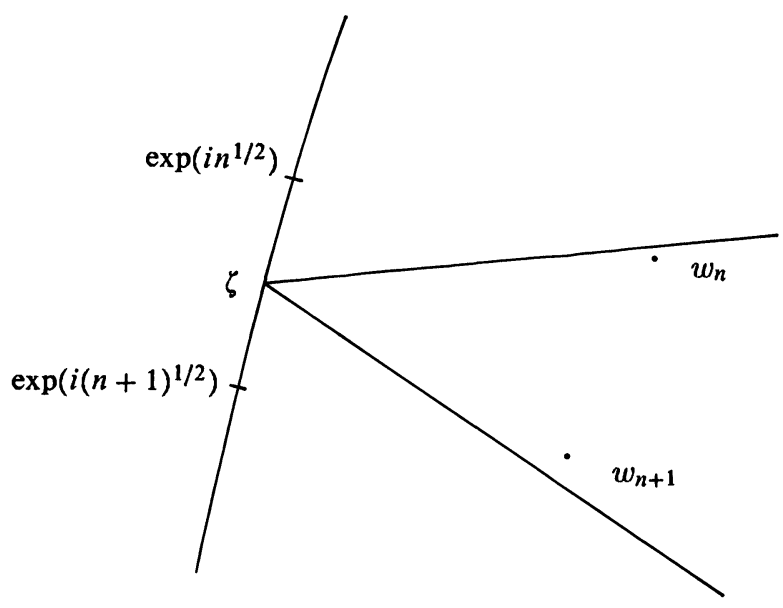

Figure 1

see this in many different ways-one is to realize that $H^{\infty}$ is closed in the weak ${ }^{*}$ topology of $L^{\infty}(\mathbf{D})$. Weak ${ }^{*}$-convergence of sequences in $H^{\infty}$ is easy to describe as bounded pointwise convergence. A sequence $u_{n} \in H^{\infty}$ converges weak ${ }^{*}$ to zero if and only if $\sup _{n}\left\|u_{n}\right\|<\infty$ and $u_{n}(\lambda) \rightarrow 0$ for every $\lambda \in \mathbf{D}$. Using this convergence one can describe a space whose dual is $H^{\infty}$. Let $\mathscr{X}$ denote the space of all linear functionals $\varphi$ in the dual of $H^{\infty}$ such that $\varphi\left(u_{n}\right) \rightarrow 0$ whenever $u_{n} \in H^{\infty}$ and $u_{n} \rightarrow 0$ weak $^{*}$. Then the dual $\mathscr{X}^{*}$ is identified naturally with $H^{\infty}$ in the following way. For every continuous functional $\Phi$ on $\mathscr{Z}$ there exists $u$ in $H^{\infty}$ such that

$$
\Phi(\varphi)=\varphi(u), \quad \varphi \in \mathscr{X} .
$$

For each $\lambda \in \mathbf{D}$ one can consider the functional $\mathscr{E}_{\lambda} \in \mathscr{Z}^{\supset}$ defined by

$$
\mathscr{E}_{\lambda}(u)=u(\lambda), \quad u \in H^{\infty} .
$$

1.5. Lemma. If $\Lambda \subset \mathbf{D}$ is dominating then the absolutely convex hull of $\left\{\mathscr{E}_{\lambda}: \lambda \in \Lambda\right\}$ is dense in the unit ball of $\mathscr{X}$.

Proof. If the conclusion were false we can find (by the HahnBanach theorem) a function $u \in H^{\infty}$ such that

$$
\begin{aligned}
& \sup \{|u(\lambda)|: \lambda \in \Lambda\}=\sup \left\{\left|\mathscr{E}_{\lambda}(u)\right|: \lambda \in \Lambda\right\} \\
& \quad<\sup \{|\varphi(u)|: \varphi \in \mathscr{X},\|\varphi\| \leq 1\}=\|u\|_{\infty} .
\end{aligned}
$$

This contradicts the assumption that $\Lambda$ is dominating. Q.E.D.

We are now ready to prove the first part of Theorem 1.2. 
1.6. Lemma. The operator $T_{w}$ in Theorem 1.2 has nonreducing invariant subspaces.

Proof. By Lemmas 1.4 and 1.5, the absolutely convex hull of $\left\{\mathscr{E}_{w_{n}}: n=1,2, \ldots\right\}$ is the unit ball of $\mathscr{X}$. Lemma 1.3 implies that we can find scalars $\left\{\alpha_{n}\right\}_{n=1}^{\infty}$ such that $\sum_{n=1}^{\infty}\left|\alpha_{n}\right|<\infty$ and $\sum_{n=1}^{\infty} \alpha_{n} \mathscr{E}_{w_{n}}=\mathscr{E}_{0}$. Define vectors $x, y \in \mathscr{H}$ by $x=\sum_{n=1}^{\infty} \alpha_{n}^{1 / 2} e_{n}$, $y=\sum_{n=1}^{\infty} \frac{\pi}{\alpha_{n}^{1 / 2}} e_{n}$ and note that we have

$$
\begin{aligned}
\left\langle T_{w}^{k} x, y\right\rangle & =\sum_{n=1}^{\infty} w_{n}^{k} \alpha_{n} \\
& =\sum_{n=1}^{\infty} \alpha_{n} \mathscr{E}_{w_{n}}\left(\lambda^{k}\right) \\
& =\mathscr{E}_{0}\left(\lambda^{k}\right)=1 \quad \text { if } k=0 \\
& =0 \quad \text { if } k \neq 0 .
\end{aligned}
$$

$(\langle\cdot, \cdot\rangle$ denotes the scalar product.) Denote by $\mathscr{M}$ the subspace generated by $\left\{x, T_{w} x, T_{w}^{2} x, \ldots\right\}$. Then $\mathscr{M}$ is invariant, $y$ is orthogonal to $T_{w} \mathscr{M}$ but not to $\mathscr{M}$ and hence $T_{w} \mid \mathscr{M}$ is not invertible. Since $T_{w}$ itself is invertible, we see that $\mathscr{M}$ is not a reducing subspace. Q.E.D.

We note here that there is nothing special about the sequence $\left\{w_{n}\right\}$ except that it is a dominating set. Also note that the full power of Lemma 1.3 has not been used in the preceding proof. For further motivation of the material to follow, let us state the following result whose proof is essentially contained in that of Lemma 1.6 (replace $\mathscr{E}_{0}$ by $\varphi$ ).

1.7. Proposition. For every functional $\varphi \in \mathscr{X}$ and every $\varepsilon>0$ there exist vectors $x, y \in \mathscr{H}$ such that $\|x\|\|y\|<\|\varphi\|+\varepsilon$ and

$$
\varphi(u)=\left\langle u\left(T_{w}\right) x, y\right\rangle
$$

for every polynomial $u$.

One could of course define $u\left(T_{w}\right)$ as the diagonal operator

$$
u\left(T_{w}\right) e_{n}=u\left(w_{n}\right) e_{n}, \quad n=1,2, \ldots
$$

for every $u \in H^{\infty}$, and then the conclusion of Proposition 1.7 would hold for any $u \in H^{\infty}$.

We proceed now to prove the second part of Theorem 1.2. 
1.8. Lemma. Every invariant subspace of $T_{w}^{-1}$ is reducing.

Proof. Let $\mathscr{M}$ be an invariant subspace for $T_{w}^{-1}$, and fix a natural number $n$. Since $w_{n}^{-1}$ is in the unbounded component of

$$
\mathbf{C} \backslash\left\{w_{m}^{-1}: m \neq n\right\}^{-},
$$

Runge's theorem implies the existence of polynomials $p_{k}$ such that $p_{k}\left(w_{n}^{-1}\right)$

$\rightarrow 1$ and $p_{k}\left(w_{m}^{-1}\right) \rightarrow 0$ uniformly in $m \neq n$. The subspace $\mathscr{M}$ is invariant under $p_{k}\left(T_{w}^{-1}\right)$, and hence under the norm limit of these operators. This limit is clearly the orthogonal projection $P_{n}$ onto the space generated by $e_{n}$. Thus $P_{n} \mathscr{M} \subset \mathscr{M}$. We conclude that for each $n$ we have either $e_{n} \in \mathscr{M}$ or $e_{n} \perp \mathscr{M}$. Therefore $\mathscr{M}$ is generated by $\left\{e_{n}: e_{n} \in \mathscr{M}\right\}$ and hence it is reducing. Q.E.D.

Returning now to the invariant subspace problem, we observe again that the existence of invariant subspaces for either $T_{w}$ or $T_{w}^{-1}$ is not an issue here-there are lots of them. Nevertheless $T_{w}$ somehow manages to have many more invariant subspaces than $T_{w}^{-1}$ (the one produced in the proof of Lemma 1.6 is just the tip of the iceberg; see [5] for more details). A moment of thought reveals two properties of $T_{w}$ that were essential in the proof:

(1) $T_{w}$ "lives" in the unit disc $\mathbf{D}$, whence its special relationship with $H^{\infty}$.

(2) The spectrum of $T_{w}$ is "rich"-the eigenvalues accumulate at all points of $\mathbf{T}$.

In some sense one can see that Theorem 1.2 is an ancestor of the following result of Brown, Chevreau and Pearcy (see [8, 12, and 10] for the original proof).

1.9. Theorem. Let $T$ be an operator on $\mathscr{H}$ such that

(i) $T$ is a contraction, i.e., $\|T\| \leq 1$; and

(ii) the spectrum $\sigma(T)$ of $T$ contains the unit circle.

Then $T$ has nontrivial invariant subspaces.

The rest of this paper will discuss in detail the proof of this theorem, starting essentially from scratch.

\section{Plan of the proof}

Throughout this section $T$ will be a fixed contraction on $\mathscr{H}$, whose spectrum contains the unit circle. 
Quasisimilarity and hyperinvariant subspaces. A subspace $\mathscr{M} \subset \mathscr{H}$ is hyperinvariant for an operator $A \in \mathscr{L}(\mathscr{H})$ if it is invariant for every operator $X$ in the commutant $\{A\}^{\prime}=\{X \in \mathscr{L}(\mathscr{H}): A X=$ $X A\}$. Two operators $A \in \mathscr{L}(\mathscr{H})$ and $A^{\prime} \in \mathscr{L}\left(\mathscr{H}^{\prime}\right)$ are quasisimilar if there exist bounded linear operators $V: \mathscr{H} \rightarrow \mathscr{H}^{\prime}$ and $W: \mathscr{H}^{\prime} \rightarrow \mathscr{H}$ such that

(i) $A^{\prime} V=V A$ and $A W=W A^{\prime}$;

(ii) $V$ and $W$ are one-to-one; and

(iii) $V$ and $W$ have dense ranges.

2.1. Lemma. Suppose $A$ and $A^{\prime}$ are quasisimilar. If $A^{\prime}$ has a nontrivial hyperinvariant subspace then $A$ has a nontrivial hyperinvariant subspace.

Proof. Let $V$ and $W$ satisfy (i)-(iii) above, and let $\mathscr{M}^{\prime}$ be a nontrivial hyperinvariant subspace for $A^{\prime}$. Define

$$
\mathscr{M}=\bigvee\left\{X W \mathscr{M}^{\prime}: X \in\{A\}^{\prime}\right\},
$$

where " $\bigvee$ " stands for "the closed linear manifold generated by." Clearly $\mathscr{M}$ is hyperinvariant and $\mathscr{M} \neq\{0\}$ because $\mathscr{M} \supset W \mathscr{M}^{\prime}$. Moreover, $\mathscr{M} \neq \mathscr{H}$ because

$$
\begin{aligned}
V \mathscr{M} & =V\left\{\bigvee X W \mathscr{M}^{\prime}: X \in\{A\}^{\prime}\right\} \\
& \subset \bigvee\left\{X^{\prime} \mathscr{M}^{\prime}: X^{\prime} \in\left\{A^{\prime}\right\}^{\prime}\right\} \\
& \subset \mathscr{M}^{\prime} \neq \mathscr{H}^{\prime}=(V \mathscr{H})^{-} .
\end{aligned}
$$

Thus $\mathscr{M}$ is nontrivial. Q.E.D.

Normal operators not of the form $\alpha I$ with $\alpha \in \mathbf{C}$ ( $I$ denotes the identity operator) are known to have nontrivial hyperinvariant subspaces provided by the spectral theorem. Therefore, in trying to prove an invariant subspace theorem, one may exclude those operators $T$ which are quasisimilar to a normal operator-these are already covered by the preceding lemma. The following result of Sz.-Nagy and Foiaş is relevant here.

2.2. Theorem. Suppose that for every $h \in \mathscr{H}, h \neq 0$, we have

$$
\lim _{n \rightarrow \infty}\left\|T^{n} h\right\| \neq 0 \neq \lim _{n \rightarrow \infty}\left\|T^{* n} h\right\| .
$$

Then $T$ is quasisimilar to a unitary operator.

Proof. The hypothesis implies that the formula

$$
\|x\|^{\prime}=\lim _{n \rightarrow \infty}\left\|T^{n} x\right\|, \quad x \in \mathscr{H},
$$


defines a norm on $\mathscr{H}$; clearly $\|x\|^{\prime} \leq\|x\|$. Denote by $\mathscr{l}^{\prime}$ the completion of $\mathscr{H}$ in the new norm, and by $X: \mathscr{H} \rightarrow \mathscr{H}^{\prime}$ the inclusion operator. We claim that there is a unitary operator $U \in$ $\mathscr{L}\left(\mathscr{H}^{\prime}\right)$ such that $U X=X T$. To prove this simply note that $\|T x\|^{\prime}=\|x\|^{\prime}, x \in \mathscr{H}$, and hence $T$ extends uniquely to an isometry $U$ on $\mathscr{H}^{\prime}$. To show that $U$ is unitary it suffices to prove that the range of $U$ is dense in $\mathscr{H}^{\prime}$, or that the range of $T$ is dense in $\mathscr{H}$. However this is immediate since the hypothesis implies that $\operatorname{ker} T^{*}=\{0\}$. Remark that $X$ is one-to-one and has dense range.

An analogous argument, with $T^{*}$ in place of $T$, proves the existence of a unitary operator $U^{\prime}$, and an operator $Z$, one-to-one and with dense range, such that $T Z=Z U^{\prime}$. To conclude the proof we show that the operator $Y=Z Z^{*} X^{*}$, which is clearly one-to-one with dense range, satisfies the relation $T Y=Y U$. Indeed, the relations $U X=X T, T Z=Z U^{\prime}, U^{-1}=U^{*}$ and $U^{\prime-1}=U^{\prime *}$ imply

$$
\begin{aligned}
T Y & =T Z Z^{*} X^{*} \\
& =Z U^{\prime} Z^{*} X^{*} \\
& =Z\left(X Z U^{\prime-1}\right)^{*} \\
& =Z\left(U^{-1} U X Z U^{\prime-1}\right)^{*} \\
& =Z\left(U^{-1} X T Z U^{\prime-1}\right)^{*} \\
& =Z\left(U^{-1} X Z U^{\prime} U^{\prime-1}\right)^{*} \\
& =Z\left(U^{-1} X Z\right)^{*} \\
& =Z Z^{*} X^{*} U \\
& =Y U . \quad \text { Q.E.D. }
\end{aligned}
$$

The preceding observations show that in our search for invariant subspaces we may assume that at least one of the spaces

$$
\mathscr{M}=\left\{x \in \mathscr{H}: \lim _{n \rightarrow \infty}\left\|T^{n} x\right\|=0\right\}
$$

and

$$
\mathscr{M}_{*}=\left\{x \in \mathscr{H}: \lim _{n \rightarrow \infty}\left\|T^{* n} x\right\|=0\right\}
$$

is not zero. It is however trivial to verify that $\mathscr{M}$ is hyperinvariant for $T$ and $\mathscr{M}_{*}$ is hyperinvariant for $T^{*}$. Thus, in fact, we may assume that either $\mathscr{M}=\mathscr{H}$ or $\mathscr{M}_{*}=\mathscr{H}$. Now, $T$ has nontrivial invariant subspaces if and only if $T^{*}$ does, so we may always 
assume that $\mathscr{M}=\mathscr{H}$ upon replacing $T$ by $T^{*}$ (if necessary). We record this assumption here for further reference.

2.3. Assumption. We have $\lim _{n \rightarrow \infty}\left\|T^{n} x\right\|=0$ for every $x \in \mathscr{H}$; in other words, $T$ is of class $C_{0}$.

Unitary dilation. Let $\mathscr{H}$ be a Hilbert space containing $\mathscr{H}$, and let $U$ be an operator acting on $\mathscr{K}$. The operator $U$ is called a dilation of $T$ if

$$
T^{n}=P_{\mathscr{H}} U^{n} \mid \mathscr{H}, \quad n=0,1,2, \ldots,
$$

where $P_{\mathscr{L}}$ denotes the orthogonal projection onto the subspace $\mathscr{H}$ of $\mathscr{K}$. If, in addition, $U$ is unitary, then we talk about a unitary dilation of $T$. A famous theorem of Sz.-Nagy says that every contraction $T$ has a unitary dilation. In the case at hand, when $T$ is assumed to be of class $C_{0}$, there is an easy proof. It starts with the observation that $I-T^{*} T$ is a positive operator-in fact

$$
\left\langle\left(I-T^{*} T\right) x, x\right\rangle=\|x\|^{2}-\|T x\|^{2}, \quad x \in \mathscr{H},
$$

and hence it has a square root $D$. We have then

$$
\|x\|^{2}-\|T x\|^{2}=\left\langle\left(I-T^{*} T\right) x, x\right\rangle=\|D x\|^{2}, \quad x \in \mathscr{H} .
$$

Thus, for $x \in \mathscr{H}$,

$$
\begin{aligned}
\|x\|^{2} & =\|x\|^{2}-\lim _{n \rightarrow \infty}\left\|T^{n} x\right\|^{2} \\
& =\sum_{n=0}^{\infty}\left(\left\|T^{n} x\right\|^{2}-\left\|T^{n+1} x\right\|^{2}\right) \\
& =\sum_{n=0}^{\infty}\left\|D T^{n} x\right\|^{2} .
\end{aligned}
$$

Let $\mathscr{D}$ denote the closure of the range of $D$, and let $\mathscr{K}$ denote the sum $\bigoplus_{k=-\infty}^{\infty} \mathscr{D}$ of infinitely many copies of $\mathscr{D}$. Define $U$ to be the bilateral shift operator on $\mathscr{K}$ :

$$
U\left(\bigoplus_{k=-\infty}^{\infty} h_{k}\right)=\bigoplus_{k=-\infty}^{\infty} h_{k-1} ;
$$

of course $U$ is unitary. Finally let $V: \mathscr{H} \rightarrow \mathscr{K}$ be defined by

$$
V h=\bigoplus_{k=-\infty}^{\infty} h_{k},
$$


where $h_{k}=D T^{-k} h$ for $k \leq 0$ and $h_{k}=0$ for $k>0$. Relation (2.4) shows that $V$ is an isometry, and a trivial calculation shows that

$$
V T^{n} h-U^{n} V h \perp V \mathscr{H}, \quad h \in \mathscr{H},
$$

and hence

$$
P_{V \mathscr{H}} U^{n} V h=V T^{n} h, h \in \mathscr{H}, \quad n=0,1,2, \ldots
$$

We conclude that $U$ is a unitary dilation of $V T V^{-1} \in \mathscr{L}(V \mathscr{H})$. Thus $U$ is a unitary dilation of $T$ if we agree to identify $\mathscr{H}$ with $V \mathscr{H}$.

The unitary dilation constructed above is a bilateral shift, and such operators are well understood. Let us give an alternate description of $U$. Consider normalized arclength measure $d \theta / 2 \pi$ on $\mathbf{T}=\left\{e^{i \theta}: 0 \leq 0<2 \pi\right\}$. The symbols $L^{p}$ will refer to $L^{p}(\mathbf{T}, d \theta / 2 \pi)$. We denote by $L^{2}(\mathscr{D})$ the space of (classes of) measurable functions $f: \mathbf{T} \rightarrow \mathscr{D}$ such that

$$
\|f\|^{2}=\frac{1}{2 \pi} \int_{0}^{2 \pi}\left\|f\left(e^{i \theta}\right)\right\|_{\mathscr{D}}^{2} d \theta<\infty .
$$

The space $L^{2}(\mathscr{D})$ can be identified with $\bigoplus_{k=-\infty}^{\infty} \mathscr{D}$ by the Fourier transform-each function $f$ in $L^{2}(\mathscr{D})$ corresponds with the sequence of its Fourier coefficients

$$
\hat{f}(n)=\frac{1}{2 \pi} \int_{0}^{2 \pi} f\left(e^{i \theta}\right) e^{-i n \theta} d \theta .
$$

Under this identification the shift $U$ becomes simply multiplication by the independent variable

$$
(U f)\left(e^{i \theta}\right)=e^{i \theta} f\left(e^{i \theta}\right), \quad \theta \in[0,2 \pi) .
$$

Summarizing, we see that the following assumption can be made about $T$.

2.5. Assumption. We have $\mathscr{H} \subset L^{2}(\mathscr{D})$ for some Hilbert space $\mathscr{D}$, and

$$
T^{n}=P_{\mathscr{H}} U^{n} \mid \mathscr{H}, \quad n=0,1,2, \ldots,
$$

where $U$ denotes the bilateral shift on $L^{2}(\mathscr{D})$.

I would like to note here that the hypothesis that $T$ is of class $C_{0}$. is not the only one that leads to Assumption 2.5. There is a more general class of contractions for which this assumption can be made-these are called the absolutely continuous contractions; 
see [5] for a more detailed discussion of this class. Much of the discussion in this article applies to all absolutely continuous contractions.

Functional calculus. Assumption 2.5 implies that

$$
p(T)=P_{\mathscr{L}} p(U) \mid \mathscr{H}
$$

for every polynomial $p$, and this suggests that we might define $p(T)$ for every function $p$ for which $p(U)$ makes sense. Now $p(U)$ makes sense for every $p \in L^{\infty}$ and, in fact,

$$
(p(U) f)(\zeta)=p(\zeta) f(\zeta), \quad p \in L^{\infty}, f \in L^{2}(\mathscr{D}),
$$

for almost every $\zeta \in \mathbf{T}$. Thus we can define $p(T)$ for every $p \in L^{\infty}$. However, the correspondence $p \rightarrow p(T)$ has the flaw that $p(T) q(T)$ is not generally equal to $(p q)(T)$ (check $p(\zeta)=\zeta$ and $q(\zeta)=1 / \zeta$ for instance). There is nevertheless a nice subalgebra of $L^{\infty}$ such that the equation

$$
(p q)(T)=p(T) q(T)
$$

is true for $p$ and $q$ in that algebra. To describe this algebra, write

$$
H^{\infty}=\left\{p \in L^{\infty}: \hat{p}(n)=0 \text { for } n<0\right\} .
$$

This is of course an abuse since $H^{\infty}$ has already been defined as the algebra of bounded analytic functions on $\mathbf{D}$. This confusion disappears if one identifies functions in $L^{\infty}$ with their sequence of Fourier coefficients, and analytic functions in $\mathbf{D}$ with the sequence of coefficients of their power series around the origin. A power series $\sum_{n=0}^{\infty} a_{n} \lambda^{n}$ defines a bounded function in $\mathbf{D}$ if and only if $\sum_{n=0}^{\infty} a_{n} e^{i n \theta}$ is the Fourier series of a bounded function on $\mathbf{T}$. The equation

$$
(p q)(T)=p(T) q(T), \quad p, q \in H^{\infty},
$$

holds because $H^{\infty}$ is the weak ${ }^{*}$ closure of polynomials in $L^{\infty}$, and (2.6) clearly holds for polynomials. Here "weak" " refers to the weak ${ }^{*}$ topology of $L^{\infty}$ as a dual space to $L^{1}$. We refer to [14 and 16] for a more detailed discussion of these points. The map $p \rightarrow p(T), p \in H^{\infty}$, is the Sz.-Nagy-Foias functional calculus.

Isometric functional calculus. A basic result of Apostol [1] is as follows; the proof will be given in $\S 3$. 
2.7. Theorem. Assume that $\sigma(T) \supset \mathbf{T}$ and the functional calculus for $T$ is not an isometry, i.e., $\|p\|_{\infty}>\|p(T)\|$ for some $p \in H^{\infty}$. Then $T$ has nontrivial hyperinvariant subspaces.

As a consequence of this result, the proof of Theorem 1.9 is reduced to the case in which $T$ satisfies the following additional assumption.

\subsection{Assumption. We have $\|p(T)\|=\|p\|_{\infty}$ for every $p \in H^{\infty}$.}

Coup de grâce. The following result was proved by Bercovici [4] and, in a somewhat weaker form, by Chevreau [11].

2.9. Theorem. Assume that the functional calculus for $T$ is an isometry. Then for every weak ${ }^{*}$-continuous functional $\varphi$ on $H^{\infty}$ and every $\varepsilon>0$ there exist vectors $x, y \in \mathscr{H}$ such that

$$
\|x\|\|y\| \leq(1+\varepsilon)\|\varphi\| \text { and } \varphi(p)=\langle p(T) x, y\rangle, \quad p \in H^{\infty} .
$$

Note that Assumption 2.3 is not an hypothesis of this theorem. However, the proof is somewhat easier under the additional assumption, and most of the ideas of the general case are present. At any rate, we saw that for the invariant subspace theorem we may assume that 2.3, 2.5 and 2.8 are true. The following result concludes the proof of Theorem 1.9.

2.10. Corollary. If the functional calculus for $T$ is an isometry, then $T$ has nontrivial invariant subspaces.

Proof. As in the proof of Lemma 1.6, choose $x, y \in \mathscr{H}$ such that $p(0)=\langle p(T) x, y\rangle, p \in H^{\infty}$, and note that $\left\langle T^{n} x, y\right\rangle=0$ for $n \geq$ 1 and $\langle x, y\rangle=1$. Two cases may arise: $T x=0$ or $T x \neq 0$. If $T x=0$ then $\operatorname{ker} T$ is a nontrivial hyperinvariant subspace for $T$. If $T x \neq 0$ then the closed linear span of $\left\{T x, T^{2} x, T^{3} x, \ldots\right\}$ is invariant for $T$, and it is nontrivial because it is orthogonal onto $y \neq 0$. Q.E.D.

\section{Isometric functional Calculus}

The main purpose of this section is to prove Apostol's Theorem 2.7. The proof will involve another (historically earlier) version of the functional calculus. It is well known that, given an operator $T$ and a function $f$ analytic on a neighborhood of the spectrum $\sigma(T)$, one can define the Riesz-Dunford functional calculus

$$
f(T)=\frac{1}{2 \pi i} \int_{\Gamma} f(\lambda)(\lambda I-T)^{-1} d \lambda,
$$


where $\Gamma$ is a simple closed curve (or a collection of such curves) surrounding $\sigma(T)$ and contained in the domain of $f$. The map $f \rightarrow f(T)$ is an algebra homomorphism, in particular $(f g)(T)=$ $f(T) g(T)$. If the spectrum of $T$ is disconnected this allows one to find invariant (indeed, hyperinvariant) subspaces for $T$. In fact, one can find in that case an analytic function $g$ which is zero on part of $\sigma(T)$ and one on another part of $\sigma(T)$. The operator $g(T)$ is a projection commuting with $T$, and its range is a nontrivial hyperinvariant subspace for $T$. One can sometimes adapt this technique for producing hyperinvariant subspaces when the spectrum of $T$ is connected.

Assume that $T$ is an operator on $\mathscr{H}$, and $\sigma(T)$ contains the unit circle T. Assume further that there exist two rectifiable simple closed curves $\Gamma$ and $\Gamma^{\prime}$ with the following properties:

(i) $\Gamma$ and $\Gamma^{\prime}$ are exterior to each other;

(ii) $\Gamma$ (resp. $\Gamma^{\prime}$ ) meets $\mathbf{T}$ at exactly two points;

(iii) $\Gamma$ and $\Gamma^{\prime}$ cross $T$ along radial segments;

(iv) $\Gamma \cap \sigma(T)=\Gamma \cap \mathbf{T}, \Gamma^{\prime} \cap \sigma(T)=\Gamma^{\prime} \cap \mathbf{T}$; and

(v) $\left\|(\lambda I-T)^{-1}\right\| \leq c /(|1-| \lambda||)$ for $\lambda$ on $\Gamma$ or $\Gamma^{\prime}$ but not on $\mathbf{T}$.

Denote $\Gamma \cap \mathbf{T}=\left\{\zeta_{1}, \zeta_{2}\right\}, \Gamma^{\prime} \cap \mathbf{T}=\left\{\zeta_{3}, \zeta_{4}\right\}$, and define operators

$$
\begin{aligned}
A & =\frac{1}{2 \pi i} \int_{\Gamma}\left(\lambda-\zeta_{1}\right)\left(\lambda-\zeta_{2}\right)(\lambda I-T)^{-1} d \lambda, \\
A^{\prime} & =\frac{1}{2 \pi i} \int_{\Gamma^{\prime}}\left(\lambda-\zeta_{3}\right)\left(\lambda-\zeta_{4}\right)(\lambda I-T)^{-1} d \lambda .
\end{aligned}
$$

Note that the two integrals are Riemann integrals because the integrands are bounded (because of $(\mathrm{v})$ ) and have at most two discontinuities (each). It is clear that $A$ commutes with $T$ and hence $(A \mathscr{H})^{-}$is an invariant subspace for $T$. In fact $A$ commutes with every $X \in\{T\}^{\prime}$ so that $(A \mathscr{H})^{-}$is hyperinvariant. To show that $(A \mathscr{H})^{-}$is not trivial we prove the following result.

3.1. Lemma. $A \neq 0, A^{\prime} \neq 0$ and $A^{\prime} A=0$.

Proof. Let $\mathscr{B} \subset \mathscr{L}(\mathscr{H})$ be a commutative Banach algebra containing $A, A^{\prime}$ and $T$. By Zorn's lemma we may also assume that $\mathscr{B}$ is maximal. Fix a point $\zeta \in \mathbf{T}$ which is surrounded by $\Gamma$. We have $\zeta \in \sigma(T)$ and therefore Gelfand theory (cf. [13]) implies that there exists a continuous algebra homomorphism $\varphi: \mathscr{B} \rightarrow \mathbf{C}$ such that $\varphi(T)=\zeta$. Now, since $\mathscr{B}$ is maximal, we have $(\lambda I-T)^{-1} \in \mathscr{B}$ whenever $\lambda \notin \sigma(T)$, and hence we can 
calculate

$$
\begin{aligned}
\varphi(A) & =\frac{1}{2 \pi i} \int_{\Gamma}\left(\lambda-\zeta_{1}\right)\left(\lambda-\zeta_{2}\right) \varphi\left((\lambda I-T)^{-1}\right) d \lambda \\
& =\frac{1}{2 \pi i} \int_{\Gamma}\left(\lambda-\zeta_{1}\right)\left(\lambda-\zeta_{2}\right)(\lambda-\zeta)^{-1} d \lambda \\
& =\left(\zeta-\zeta_{1}\right)\left(\zeta-\zeta_{2}\right)
\end{aligned}
$$

by the Cauchy theorem. Since $\varphi(A) \neq 0$, we must have $A \neq 0$. The proof that $A^{\prime} \neq 0$ is virtually identical.

The proof that $A^{\prime} A=0$ uses a standard technique from the Riesz-Dunford functional calculus. In the calculation to follow we will use the Cauchy theorem along with the easily verified "resolvent equation"

$$
\frac{1}{\mu-\lambda}\left[(\lambda I-T)^{-1}-(\mu I-T)^{-1}\right]=(\lambda I-T)^{-1}(\mu I-T)^{-1} .
$$

With the notation $p(\lambda)=\left(\lambda-\zeta_{1}\right)\left(\lambda-\zeta_{2}\right), q(\mu)=\left(\mu-\zeta_{3}\right)\left(\mu-\zeta_{4}\right)$ we have

$$
\begin{aligned}
A^{\prime} A= & \frac{1}{(2 \pi i)^{2}} \int_{\Gamma^{\prime}} \int_{\Gamma} p(\lambda) q(\mu)(\lambda I-T)^{-1}(\mu I-T)^{-1} d \lambda d \mu \\
= & \frac{1}{(2 \pi i)^{2}} \int_{\Gamma^{\prime}} \int_{\Gamma} \frac{p(\lambda) q(\mu)}{\mu-\lambda}\left[(\lambda I-T)^{-1}-(\mu I-T)^{-1}\right] d \lambda d \mu \\
= & \frac{1}{(2 \pi i)^{2}} \int_{\Gamma} p(\lambda)(\lambda I-T)^{-1}\left[\int_{\Gamma^{\prime}} \frac{q(\mu)}{\mu-\lambda} d \mu\right] d \lambda \\
& -\frac{1}{(2 \pi i)^{2}} \int_{\Gamma^{\prime}} q(\mu)(\mu I-T)^{-1}\left[\int_{\Gamma} \frac{p(\lambda)}{\mu-\lambda} d \lambda\right] d \mu,
\end{aligned}
$$

and the integrals in square brackets are zero by the Cauchy theorem. Q.E.D.

For the remaining part of this section $T$ will denote a contraction operator such that $\sigma(T) \supset \mathbf{T}$. We see from the above argument that to find a nontrivial hyperinvariant subspace it suffices to find curves $\Gamma$ and $\Gamma^{\prime}$ with properties (i)-(v) above. We note first that the inequality in (v) is true for any $\lambda$ with $|\lambda|>1$. Indeed, in that case the fact that the Sz.-Nagy-Foias functional calculus is contractive implies

$$
\begin{aligned}
\left\|(\lambda I-T)^{-1}\right\| & \leq \sup \left\{\frac{1}{|\lambda-\mu|}: \mu \in \mathbf{D}\right\} \\
& =\frac{1}{\operatorname{dist}(\lambda, \mathbf{D})}=\frac{1}{|\lambda|-1} .
\end{aligned}
$$


One must concentrate therefore on the portion of $\Gamma$ and $\Gamma^{\prime}$ that are contained in $\mathbf{D}$. Let us set

$$
\Lambda=(\sigma(T) \cap \mathbf{D}) \cup\left\{\lambda \in \mathbf{D} \backslash \sigma(T):\left\|(\lambda I-T)^{-1}\right\| \geq \frac{3}{1-|\lambda|}\right\},
$$

and denote by $\omega$ the set of those $\zeta \in \mathbf{T}$ which are not radial limits of points in $\Lambda$.

3.2. Lemma. If $\omega$ is uncountable then $T$ has nontrivial hyperinvariant subspaces.

Proof. For each $\zeta \in \omega$ choose two rational numbers $a_{\zeta}, b_{\zeta} \in$ $(0,1)$ such that the set $A_{\zeta}=\left\{t \zeta: a_{\zeta}<t<1\right\} \cup\left\{\lambda:\left|\lambda-a_{\zeta} \zeta\right|<b_{\zeta}\right\}$ is contained in $\mathbf{D} \backslash \Lambda$. Since $\omega$ is not countable there are $a, b \in$ $(0,1)$ and an uncountable $\omega^{\prime} \subset \omega$ such that $a_{\zeta}=a$ and $b_{\zeta}=b$ for all $\zeta \in \omega^{\prime}$. Clearly then we can find $\zeta_{1}, \zeta_{2}, \zeta_{3}, \zeta_{4} \in \omega^{\prime}$ such that $A_{\zeta_{1}} \cap A_{\zeta_{2}} \cap A_{\zeta_{3}} \cap A_{\zeta_{4}} \neq 0$. Supposing that the $\zeta_{j}$ are arranged in counter clockwise order one can construct $\Gamma$ and $\Gamma^{\prime}$ satisfying (i)-(v) such that $\Gamma \cap \mathbf{D} \subset A_{\zeta_{1}} \cup A_{\zeta_{2}}$ and $\Gamma^{\prime} \cap \mathbf{D} \subset A_{\zeta_{3}} \cup A_{\zeta_{4}}$. Q.E.D.

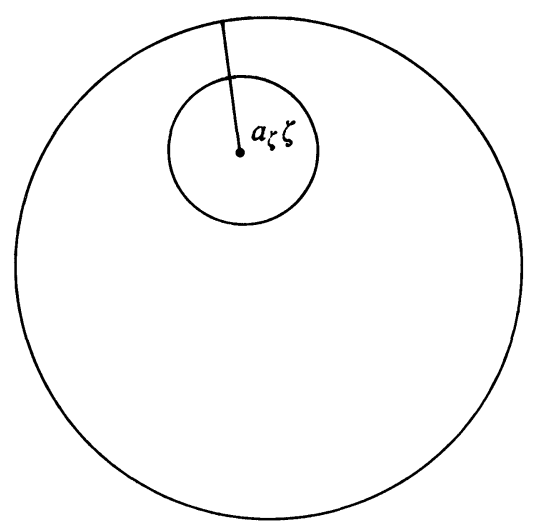

FIGURE 2

3.3. Theorem. If $\Lambda$ is not dominating then $T$ has nontrivial hyperinvariant subspaces. If $\Lambda$ is dominating then the Sz.-Nagy-Foias functional calculus is isometric.

Proof. The first part of the statement follows from Lemma 2.2, so we proceed to the second part. Assume therefore that $\Lambda$ is dominating and fix $u \in H^{\infty}$ and $\lambda \in \Lambda$. We can find $v \in H^{\infty}$ such that

$$
u(z)-u(\lambda)=(\lambda-z) v(z), \quad z \in \mathbf{D},
$$


and $\|v\|_{\infty} \leq 2\|u\|_{\infty} /(1-|\lambda|)$. We clearly have

$$
u(T)-u(\lambda) I=(\lambda I-T) v(T)=v(T)(\lambda I-T) .
$$

If $\lambda \in \sigma(T)$ it follows that $u(\lambda) \in \sigma(u(T))$ and hence

$$
\|u(T)\| \geq|u(\lambda)|, \quad \lambda \in \sigma(T) \cap \Lambda .
$$

If $\lambda \in \Lambda \backslash \sigma(T)$ then we can choose a vector $\xi \in \mathscr{H},\|\xi\|=1$, such that

$$
\|(\lambda I-T) \xi\| \leq \frac{1}{3}(1-|\lambda|) .
$$

We have

$$
\begin{aligned}
\|u(T)\| & \geq\|u(T) \xi\| \\
& \geq|u(\lambda)|-\|(u(T)-u(\lambda) I) \xi\| \\
& \geq|u(\lambda)|-\|v\|_{\infty}\|(\lambda I-T) \xi\| \\
& \geq|u(\lambda)|-\frac{2}{3}\|u\|_{\infty},
\end{aligned}
$$

and hence

$$
\|u(T)\|+\frac{2}{3}\|u\|_{\infty} \geq|u(\lambda)|, \quad \lambda \in \Lambda \backslash \sigma(T) .
$$

Relations (3.4) and (3.5) show now that

$$
\|u(T)\|+\frac{2}{3}\|u\|_{\infty} \geq \sup \{|u(\lambda)|: \lambda \in \Lambda\}=\|u\|_{\infty}
$$

because $\Lambda$ is dominating. Thus $\|u(T)\| \geq \frac{1}{3}\|u\|_{\infty}$. Applying this inequality to $u^{n}$ we get

$$
\|u(T)\| \geq\left\|u(T)^{n}\right\|^{1 / n} \geq\left(\frac{1}{3}\left\|u^{n}\right\|_{\infty}\right)^{1 / n}=\left(\frac{1}{3}\right)^{1 / n}\|u\|_{\infty},
$$

whence $\|u(T)\| \geq\|u\|_{\infty}$. The opposite inequality $\|u(T)\| \leq\|u\|_{\infty}$ is always true. Q.E.D.

We conclude this section with a result about essential norms and some consequences that are important for the developments to follow. We recall that the attribute "essential" refers to concepts related with the quotient algebra $\mathscr{L}(\mathscr{H}) / \mathscr{K}(\mathscr{H})$, where $\mathscr{K}(\mathscr{H})$ is the ideal of compact operators on $\mathscr{H}$. Thus, the essential spectrum $\sigma_{e}(T)$ is the spectrum of the class of $T$ in this quotient algebra, and the essential norm $\|T\|_{e}$ is the norm of the class of $T$. We always have $\sigma_{e}(T) \subset \sigma(T)$ and $\|T\|_{e} \leq\|T\|$.

3.6. Proposition. Assume $T$ satisfies Assumption 2.3 and that the functional calculus for $T$ is isometric. Then in fact $\|u(T)\|_{e}=$ $\|u\|_{\infty}$ for every $u \in H^{\infty}$.

Proof. Fix $u \in H^{\infty}$ and note that the last part of the proof of Theorem 3.3 shows that $\|u(T)\|$ coincides with the spectral radius 
of $u(T)$. Assume, to get a contradiction, that $\|u(T)\|>\|u(T)\|_{e}$. We conclude that there is an eigenvalue $\mu$ for $u(T)$, with finite multiplicity, with $|\mu|=\|u\|_{\infty}$. The space $\mathscr{M}=\operatorname{ker}(u(T)-\mu I)$ is invariant for $T$, hence $T \mid \mathscr{M}$ has an eigenvalue $\lambda$. Since $T$ is of class $C_{0}$ (Assumption 2.3), we must have $|\lambda|<1$, and therefore $\operatorname{ker}(T-\lambda I) \subset \operatorname{ker}(u(T)-u(\lambda) I)$. We deduce that $\mu=u(\lambda)$, and hence $u$ attains its maximum. It follows that $u$ is constant and in that case clearly $\|u(T)\|=\|u(T)\|_{e}$, a contradiction. Q.E.D.

From this point on $T$ will be assumed to satisfy Assumptions 2.3, 2.5 and 2.8. Thus, in particular, $\mathscr{H} \subset L^{2}(\mathscr{D})$. We denote by $\chi_{\omega}$ the characteristic function of the set $\omega$. Proposition 3.6 will allow us to find functions in $\mathscr{H}$ which are "concentrated" on a given subset of $\mathbf{T}$.

3.7. Lemma. Given a subset $\omega \subset \mathbf{T}$ with positive Lebesgue measure, a natural number $p, \varepsilon>0$, and vectors $\xi_{1}, \xi_{2}, \ldots, \xi_{p} \in$ $L^{2}(\mathscr{D})$, we can find a vector $x \in \mathscr{H}, x \neq 0$, such that

(i) $\left\|\chi_{\mathbf{T} \backslash \omega} x\right\|<\varepsilon\left\|\chi_{\omega} x\right\|$; and

(ii) $\left\langle x, \xi_{j}\right\rangle=0, j=1,2, \ldots, p$.

Proof. Choose $\delta>0$ such that $\delta /(1-2 \delta)<\varepsilon^{2}$, and fix a function $u \in H^{\infty}$ such that $|u(\zeta)|=1$ for almost every $\zeta \in \omega$, and $|u(\zeta)|=\delta^{1 / 2}$ for almost every $\zeta \in \mathbf{T} \backslash \omega$. Proposition 3.6 implies the existence of $x \in \mathscr{H}$ such that $\left\langle x, \xi_{j}\right\rangle=0,1 \leq j \leq p$, $\|x\|=1$, and $\|u(T) x\|>(1-\delta)^{1 / 2}$. But then we also have $\|u x\|=\|u(U) x\| \geq\|u(T) x\|>(1-\delta)^{1 / 2}$. Thus

$$
\begin{aligned}
\left\|\chi_{\omega} x\right\|^{2}+\left\|\chi_{\mathbf{T} \backslash \omega} x\right\|^{2}-\delta & =1-\delta \\
& <\|u x\|^{2} \\
& =\left\|\chi_{\omega} x\right\|^{2}+\delta\left\|\chi_{\mathbf{T} \backslash \omega} x\right\|^{2},
\end{aligned}
$$

from which we infer $\left\|\chi_{T \backslash \omega} x\right\|^{2}<\delta /(1-\delta)$. We have then $\left\|\chi_{\omega} x\right\|^{2}$ $=1-\left\|\chi_{\mathbf{T} \backslash \omega} x\right\|^{2}>(1-2 \delta) /(1-\delta)$ so that

$\left\|\chi_{\mathbf{T} \backslash \omega} x\right\|^{2}<\frac{\delta}{1-2 \delta} \cdot \frac{1-2 \delta}{1-\delta}<\frac{\delta}{1-2 \delta}\left\|\chi_{\omega} x\right\|^{2}<\varepsilon^{2}\left\|\chi_{\omega} x\right\|^{2}$. Q.E.D.

We need the following slightly different version of this result.

3.8. Proposition. Let $f \in L^{\infty}$ be a function such that $0 \leq f \leq 1$, let $\xi_{1}, \xi_{2}, \ldots, \xi_{p} \in L^{2}(\mathscr{D})$ and $\varepsilon \in(0,1)$. If $\|f\|_{\infty}>1-\varepsilon$, 
there exists $x \in \mathscr{H}, x \neq 0$, such that $\left\langle x, \xi_{j}\right\rangle=0,1 \leq j \leq p$, and $\left\|(1-f)^{1 / 2} x\right\|^{2}<(\varepsilon /(1-\varepsilon))\left\|f^{1 / 2} x\right\|^{2}$.

Proof. Choose $\varepsilon^{\prime}<\varepsilon$ such that the set $\omega=\left\{\zeta: f(\zeta)>1-\varepsilon^{\prime}\right\}$ has positive measure, and choose $\delta>0$ such that

$$
\frac{\varepsilon^{\prime}+\delta^{2}}{1-\varepsilon^{\prime}}<\frac{\varepsilon}{1-\varepsilon} \text {. }
$$

By Lemma 3.7 we can find $x \in \mathscr{H}, x \neq 0$, with $\left\langle x, \xi_{j}\right\rangle=0$, $1 \leq j \leq p$, and $\left\|\chi_{\mathbf{T} \backslash \omega} x\right\|^{2}<\delta\left\|\chi_{\omega} x\right\|^{2}$. We will show that this $x$ satisfies the conclusion of our proposition. We have

$$
\begin{aligned}
\left\|f^{1 / 2} x\right\|^{2} & \geq \frac{1}{2 \pi} \int_{\omega} f\left(e^{i \theta}\right)\left\|x\left(e^{i \theta}\right)\right\|^{2} d \theta \\
& \geq\left(1-\varepsilon^{\prime}\right)\left\|\chi_{\omega} x\right\|^{2},
\end{aligned}
$$

and hence

$$
\begin{aligned}
\left\|(1-f)^{1 / 2} x\right\|^{2}= & \frac{1}{2 \pi} \int_{\omega}\left(1-f\left(e^{i \theta}\right)\right)\left\|x\left(e^{i \theta}\right)\right\|^{2} d \theta \\
& +\frac{1}{2 \pi} \int_{T \backslash \omega}\left(1-f\left(e^{i \theta}\right)\right)\left\|x\left(e^{i \theta}\right)\right\|^{2} d \theta \\
\leq & \varepsilon^{\prime}\left\|\chi_{\omega} x\right\|^{2}+\left\|\chi_{T \backslash \omega} x\right\|^{2} \\
& <\varepsilon^{\prime}\left\|\chi_{\omega} x\right\|^{2}+\delta^{2}\left\|\chi_{\omega} x\right\|^{2} \\
& <\frac{\varepsilon^{\prime}+\delta^{2}}{1-\varepsilon^{\prime}}\left\|f^{1 / 2} x\right\|^{2} \\
& \leq \frac{\varepsilon}{1-\varepsilon}\left\|f^{1 / 2} x\right\|^{2} \text {. Q.E.D. }
\end{aligned}
$$

4. BOUNDED FUNCTIONS AND THE STRUCTURE OF THE UNITARY DILATION

Throughout this section $T$ will be a contraction operator subject to Assumptions 2.3, 2.5 and 2.8. We will denote by $L^{\infty}(\mathscr{D})$ the set of (classes of) bounded functions in $L^{2}(\mathscr{D})$. The main purpose of this section is to show that $L^{\infty}(\mathscr{D}) \cap \mathscr{H}$ is dense in $\mathscr{H}$. Along the way we develop some useful facts related to the embedding of $\mathscr{H}$ in $L^{2}(\mathscr{D})$.

Let us denote, as before, $L^{1}=L^{1}(\mathbf{T}, d \theta / 2 \pi)$. For any pair of vectors $x, y \in L^{2}(\mathscr{D})$ we can define a function $x \cdot y \in L^{1}$ by setting

$$
(x \cdot y)(\zeta)=\langle x(\zeta), y(\zeta)\rangle
$$


for almost every $\zeta \in \mathbf{T}$; the scalar product on the right-hand side is calculated in $\mathscr{D}$. Clearly $x$ is essentially bounded if and only if $x \cdot x$ is essentially bounded. We note for further use that the Fourier coefficients $(x \cdot y)^{\wedge}(n)$ of $x \cdot y$ can be calculated as follows

$$
(x \cdot y)^{\wedge}(n)=\left\langle U^{-n} x, y\right\rangle, \quad n \in \mathbf{Z}, x, y \in L^{2}(\mathscr{D}),
$$

and

$$
\begin{aligned}
(x \cdot y)^{\wedge}(n) & =\left\langle T^{-n} x, y\right\rangle, & & n \leq 0 \\
& =\left\langle T^{* n} x, y\right\rangle, & & n>0,
\end{aligned}
$$

if $x, y \in \mathscr{H}$. Formula (4.1) is immediate.

$$
\begin{aligned}
(x \cdot y)^{\wedge}(n) & =\frac{1}{2 \pi} \int_{0}^{2 \pi} e^{-i n \theta}(x \cdot y)\left(e^{i \theta}\right) d \theta \\
& =\frac{1}{2 \pi} \int_{0}^{2 \pi}\left\langle e^{-i n \theta} x\left(e^{i \theta}\right), y\left(e^{i \theta}\right)\right\rangle d \theta \\
& =\left\langle U^{-n} x, y\right\rangle
\end{aligned}
$$

by the definition of the scalar product in $L^{2}(\mathscr{D})$, and (4.2) follows because $U$ is a dilation of $T$ and hence for $x, y \in \mathscr{H}$ and $n \geq 0$

$$
\begin{aligned}
\left\langle U^{n} x, y\right\rangle & =\left\langle U^{n} x, P_{\mathscr{H}} y\right\rangle \\
& =\left\langle P_{\mathscr{H}} U^{n} x, y\right\rangle \\
& =\left\langle T^{n} x, y\right\rangle .
\end{aligned}
$$

A subspace $\mathscr{W} \subset L^{2}(\mathscr{D})$ is called a wandering space if the spaces $\left\{U^{n} \mathscr{W}: n \in \mathbf{Z}\right\}$ are pairwise orthogonal. One can verify without difficulty that $\mathscr{W}$ is wandering if and only if $\mathscr{W} \perp U^{n} \mathscr{W}$ for $n=1,2,3, \ldots$. Moreover, if $\mathscr{W}$ is wandering then so is $U^{n} \mathscr{W}$ for any $n \in \mathbf{Z}$.

4.3. Lemma. Every vector in a wandering space is bounded.

Proof. Let $x$ be an element of a wandering space. It follows from (4.1) that $(x \cdot x)^{\wedge}(n)=0$ for all $n \neq 0$, and hence $x \cdot x$ is essentially constant, hence bounded. Q.E.D.

4.4. Lemma. (i) The spaces

$$
\mathscr{V}=\{U h-T h: h \in \mathscr{H}\}^{-} \text {and } \mathscr{W}=\left\{U^{*} h-T^{*} h: h \in \mathscr{H}\right\}^{-}
$$

are wandering.

(ii) For every $x \in \mathscr{H}$ and $n=1,2, \ldots$ we have

$$
U^{* n} x-T^{* n} x \in \mathscr{W} \oplus U^{*} \mathscr{W} \oplus \cdots \oplus U^{* n-1} \mathscr{W}
$$

and hence $U^{* n} x-T^{* n} x$ is bounded. 
Proof. (i) We only prove that $\mathscr{V}$ is wandering; $\mathscr{W}$ is treated analogously. It suffices to show that $U h-T h$ and $U^{n}(U k-T k)$ are orthogonal for $n \geq 1$ and $h, k \in \mathscr{H}$. Indeed,

$$
\begin{aligned}
& \left\langle U h-T h, U^{n}(U k-T k)\right\rangle \\
& \quad=\left\langle U h, U^{n+1} k\right\rangle-\left\langle U h, U^{n} T k\right\rangle-\left\langle T h, U^{n+1} k\right\rangle+\left\langle T h, U^{n} T k\right\rangle \\
& \quad=\left\langle h, U^{n} k\right\rangle-\left\langle h, U^{n-1} T k\right\rangle-\left\langle T h, U^{n+1} k\right\rangle+\left\langle T h, U^{n} T k\right\rangle \\
& \quad=\left\langle h, T^{n} k\right\rangle-\left\langle h, T^{n-1} T k\right\rangle-\left\langle T h, T^{n+1} k\right\rangle+\left\langle T h, T^{n} T k\right\rangle \\
& \quad=0,
\end{aligned}
$$

where we used the fact that $U$ is unitary and dilates $T$.

$$
\begin{aligned}
U^{* n} x-T^{* n} x & =\sum_{k=0}^{n-1}\left(U^{* n-k} T^{* k} x-U^{* n-k-1} T^{* k+1} x\right) \\
& =\sum_{k=0}^{n-1} U^{* n-k-1}\left(U^{*} y_{k}-T^{*} y_{k}\right),
\end{aligned}
$$

where $y_{k}=T^{* k} x$. Q.E.D.

Finally we use the fact that $T$ is of class $C_{0}$. (Assumption 2.3) to prove that bounded functions are dense in $\mathscr{H}$.

4.5. Proposition. (i) $\mathscr{H} \subset \bigoplus_{n=1}^{\infty} U^{* n} \mathscr{V}$.

(ii) $\mathscr{H} \cap L^{\infty}(\mathscr{D})$ is dense in $\mathscr{H}$.

Proof. Fix $x \in \mathscr{H}$ and note that

$$
\begin{aligned}
x & =x-\lim _{n \rightarrow \infty} U^{* n} T^{n} x \\
& =\sum_{n=0}^{\infty}\left(U^{* n} T^{n} x-U^{* n+1} T^{n+1} x\right) \\
& =\sum_{n=0}^{\infty} U^{* n+1}\left(U y_{n}-T y_{n}\right),
\end{aligned}
$$

where $y_{n}=T^{n} x$. Thus (i) follows. To prove (ii) it suffices to show that $P_{\mathscr{H}}\left(U^{* n+1}(U y-T y)\right)$ is bounded for every $y \in \mathscr{H}$ and $n \geq 0$. Since $U$ is a dilation of $T$, we have

$$
\begin{aligned}
P_{\mathscr{H}}\left(U^{* n+1}(U y-T y)\right) & =P_{\mathscr{C}}\left(U^{* n} y-U^{* n+1} T y\right) \\
& =T^{* n} y-T^{* n+1} T y .
\end{aligned}
$$


Now, $U^{* n+1}(U y-T y)$ is bounded as an element of a wandering space, and therefore it suffices to show that $T^{* n} y-T^{* n+1} y-$ $U^{* n+1}(U y-T y)$ is bounded. This however is clear from Lemma 4.4(ii). Q.E.D.

The most important consequence for us of this proposition is the following improvement of Proposition 3.8 (the improvement consists of the fact that $z$ is bounded).

4.6. Proposition. Let $f \in L^{\infty}$ be a function such that $0 \leq f \leq 1$, let $\xi_{1}, \xi_{2}, \ldots, \xi_{p} \in L^{2}(\mathscr{D})$ and $\varepsilon \in(0,1)$. If $\|f\|_{\infty}>1-\varepsilon$, there exists a bounded function $z \in \mathscr{H}, z \neq 0$, such that $\left\langle z, \xi_{j}\right\rangle=0$, $1 \leq j \leq p$, and $\left\|(1-f)^{1 / 2} z\right\|^{2}<(\varepsilon /(1-\varepsilon))\left\|f^{1 / 2} z\right\|^{2}$.

This version is an immediate consequence of Proposition 3.8we just approximate the vector $x$ of that proposition with a bounded function. One only needs to note the following elementary lemma, whose proof is left as an exercise.

4.7. Lemma. Let $\mathscr{H}$ be a Hilbert space, $\mathscr{M}$ a dense linear manifold in $\mathscr{H}$, and $\mathscr{H}^{\prime}$ a finite-codimensional subspace of $\mathscr{H}$. Then $\mathscr{M} \cap \mathscr{H}^{\prime}$ is dense in $\mathscr{H}^{\prime}$.

For the proof of Proposition 4.6 take

$$
\mathscr{H}^{\prime}=\left\{x \in \mathscr{H}:\left\langle x, \xi_{j}\right\rangle=0,1 \leq j \leq p\right\} .
$$

\section{WEAK ${ }^{*}$-CONTINUOUS FUNCTIONALS ON $H^{\infty}$}

In this section we will reformulate Theorem 2.8 in the form in which we prove it. All the assumptions and notation of $\S 4$ remain in force here. Let $H_{0}^{1}$ denote the space of those (classes of) functions $f \in L^{1}$ for which $\hat{f}(n)=0, n \leq 0$. The quotient $L^{1} / H_{0}^{1}$ is a Banach space, and we will denote by $[f]$ the class (coset) in this space of a function $f \in L^{1}$. We will argue below that the following statement is equivalent to Theorem 2.8 .

5.1. Theorem. For every $\varphi \in L^{1} / H_{0}^{1}$ and every $\varepsilon>0$ there exist vectors $x, y \in \mathscr{H}$ such that $\|x\|\|y\| \leq(1+\varepsilon)\|\varphi\|$ and $\varphi=[x \cdot y]$.

The product $x \cdot y$ for $x, y \in L^{2}(\mathscr{D})$ was defined in $\S 4$. Let us note that this statement does imply the existence of invariant subspaces for $T$. Indeed, if we choose $\varphi=[1]$ (the class of the constant one function) the equality $[x \cdot y]=\varphi$ means that 
$(x \cdot y)^{\wedge}(n)=\hat{1}(n)$ for $n \leq 0$ or $($ cf. (4.2)) that

$$
\begin{aligned}
\left\langle T^{n} x, y\right\rangle & =1 & & \text { if } n=0 \\
& =0 & & \text { if } n \neq 0 .
\end{aligned}
$$

The fact that $T$ has nontrivial invariant subspaces follows now as in Corollary 2.9.

Theorems 2.8 and 5.1 are truly identical because there is an isometry that associates with every element $\varphi$ of $L^{1} / H_{0}^{1}$ an element $\psi$ of the space $\mathscr{X}($ see $\S 1)$ in such a way that $[x \cdot y]=\varphi$ if and only if

$$
\langle u(T) x, y\rangle=\psi(u), \quad u \in H^{\infty} .
$$

We will not dwell on this subject any longer since it is somewhat technical, and since the invariant subspace result follows from Theorem 5.1 .

\section{FUNCTIONS CONCENTRATED ON A GIVEN SET}

The remainder of this paper is dedicated to the proof of Theorem 5.1 (under Assumptions 2.3, 2.5 and 2.8). It is interesting to note that the central fact we use is Proposition 4.6. The structure of the contraction $T$ disappears from the stage. Proposition 4.6 tells us about the existence of bounded functions in $\mathscr{H}$ that are concentrated on a set. In this section we will further study these functions showing that they can be controlled very well in some sense. This presentation contains ideas from [8] and [3].

Fix a set $\sigma \subset \mathbf{T}$ with positive measure $|\sigma|$, vectors $\xi_{1}, \xi_{2}, \ldots$, $\xi_{p} \in L^{2}(\mathscr{D})$, and two positive numbers $\eta$ and $\delta$.

6.1. Definition. . The set $S\left(\sigma ; \xi_{1}, \xi_{2}, \ldots, \xi_{p} ; \eta, \delta\right)$ consists of those vectors $x \in \mathscr{H}$ such that

(a) $\left\langle x, \xi_{j}\right\rangle=0,1 \leq j \leq p$;

(b) $x$ can be written as $x=g+b, g, b \in L^{2}(\mathscr{D})$; and

(b1) $\|g(\zeta)\| \leq \chi_{\sigma}(\zeta)$ for almost every $\zeta \in \mathbf{T}$;

(b2) $\|b\|<\eta\|g\|$;

(b3) $\left|\left\langle g, \xi_{j}\right\rangle\right|<\delta\|x\|, 1 \leq j \leq p$.

Observe that Proposition 4.6 guarantees the existence of nonzero elements in $S\left(\sigma ; \xi_{1}, \xi_{2}, \ldots, \xi_{p} ; \eta ; \delta\right)$. Indeed, let $f=\chi_{\sigma}$, and let $z$ be given by that proposition. Choose $\alpha>0$ small enough that $\alpha\|z(\zeta)\| \leq 1$ almost everywhere, and set $x=\alpha z, g=\chi_{\sigma} x$, $b=\chi_{\mathbf{T} \backslash \sigma} x$. Properties (a), (b1) and (b2) are immediate if $\varepsilon$ is 
small enough. For (b3) we note that

$$
\left|\left\langle g, \xi_{j}\right\rangle\right|=\left|\left\langle b, \xi_{j}\right\rangle\right| \leq\|b\|\left\|\xi_{j}\right\| \leq\left(\frac{\varepsilon}{1-\varepsilon}\right)^{1 / 2}\left\|\xi_{j}\right\|\|g\|
$$

so that (b3) is also verified if $\varepsilon$ is small enough.

If $x=g+b \in S\left(\sigma ; \xi_{1}, \xi_{2}, \ldots, \xi_{p} ; \eta ; \delta\right)$ then clearly

$$
\frac{\|x\|}{1+\eta} \leq\|g\| \leq \frac{\|x\|}{1-\eta} \text {. }
$$

6.2. Proposition. If $\eta<\frac{1}{2}$ then

$$
\sup \left\{\|x\|: x \in S\left(\sigma ; \xi_{1}, \xi_{2}, \ldots, \xi_{p} ; \eta ; \delta\right)\right\}>2^{-3} \eta|\sigma|^{1 / 2} .
$$

Proof. For simplicity we write $S=S\left(\sigma ; \xi_{1}, \xi_{2}, \ldots, \xi_{p} ; \eta ; \delta\right)$, and let us set $\sup \{\|x\|: x \in S\}=\gamma|\sigma|^{1 / 2}$. Assume, to get a contradiction, that $\gamma \leq 2^{-3} \eta$. Let $x_{n} \in S$ be a sequence such that $\lim _{n \rightarrow \infty}\left\|x_{n}\right\|=\gamma|\sigma|^{1 / 2}$, and write $x_{n}=g_{n}+b_{n}$, as required by the definition of the set $S$. Note that

$$
\left\|g_{n}\right\| \leq\left\|x_{n}\right\| /(1-\eta) \leq 2^{-2} \eta|\sigma|^{1 / 2},
$$

and set

$$
\omega_{n}=\left\{\zeta \in \sigma:\left\|g_{n}(\zeta)\right\|<\frac{1}{2}\right\}
$$

Then

$$
\begin{aligned}
\left|\sigma \backslash \omega_{n}\right| & =\frac{1}{2 \pi} \int_{\sigma \backslash \omega_{n}} d \theta \\
& \leq 4 \cdot \frac{1}{2 \pi} \int_{\sigma \backslash \omega_{n}}\left\|g_{n}\left(e^{i \theta}\right)\right\|^{2} d \theta \\
& \leq 4\left\|g_{n}\right\|^{2} \\
& \leq 2^{-2} \eta^{2}|\sigma|,
\end{aligned}
$$

and hence

$$
\left|\omega_{n}\right|=\frac{1}{2 \pi} \int_{0}^{2 \pi} \chi_{\omega_{n}}\left(e^{i \theta}\right) d \theta \geq\left(1-2^{-2} \eta^{2}\right)|\sigma| .
$$

Dropping to a subsequence we may assume that

(i) $x_{n}$ converge weakly in $\mathscr{H}$ to $x$;

(ii) $\chi_{\omega_{n}} g_{n}$ converge weakly in $L^{2}(\mathscr{D})$ to $u$;

(iii) $\chi_{\mathbf{T} \backslash \omega_{n}} b_{n}$ converge weakly in $L^{2}(\mathscr{D})$ to $v$; and

(iv) $\chi_{\omega_{n}}$ converge weak ${ }^{*}$ in $L^{\infty}$ to $f, 0 \leq f \leq \chi_{\sigma}$. 
Note that

$$
\frac{1}{2 \pi} \int_{0}^{2 \pi} f\left(e^{i \theta}\right) d \theta=\lim _{n \rightarrow \infty} \frac{1}{2 \pi} \int_{0}^{2 \pi} \chi_{\omega_{n}}\left(e^{i \theta}\right) d \theta \geq\left(1-2^{-2} \eta^{2}\right)|\sigma|,
$$

and hence we must have $f(\zeta)>1-2^{-1} \eta^{2}$ for $\zeta$ in a set of positive measure. An application of Proposition 4.6 yields a bounded function $z \in \mathscr{H}, z \neq 0$, such that

(v) $\langle z, u\rangle=\langle z, v\rangle=\langle z, x\rangle=\left\langle z, \xi_{j}\right\rangle=\left\langle z, f \xi_{j}\right\rangle=0$ for $1 \leq j \leq p$; and

(vi) $\left\|(1-f)^{1 / 2} z\right\|<\left(2^{-1} \eta^{2} /\left(1-2^{-1} \eta^{2}\right)\right)\left\|f^{1 / 2} z\right\|^{2}<\eta^{2}\left\|f^{1 / 2} z\right\|^{2}$. Dividing $z$ by a sufficiently large constant we may assume that $\|z(\zeta)\| \leq \frac{1}{2}$ for almost every $\zeta \in \mathbf{T}$. We claim that for sufficiently large $n$ the vector $x_{n}^{\prime}=x_{n}+z$ belongs to $S$ and $\left\|x_{n}^{\prime}\right\|>\gamma|\sigma|^{1 / 2}$. The latter inequality is verified because

$$
\begin{aligned}
\lim _{n \rightarrow \infty}\left\|x_{n}^{\prime}\right\|^{2} & =\lim _{n \rightarrow \infty}\left(\left\|x_{n}\right\|^{2}+\|z\|^{2}+2 \operatorname{Re}\left\langle x_{n}, z\right\rangle\right) \\
& =\gamma^{2}|\sigma|+\|z\|^{2}+2 \operatorname{Re}\langle x, z\rangle \\
& =\gamma^{2}|\sigma|+\|z\|^{2} \\
& >\gamma^{2}|\sigma|
\end{aligned}
$$

here we used (i) and (v). To see that $x_{n}^{\prime} \in S$ we note first that $x_{n}^{\prime}$ satisfies condition (a) of the definition by (v). Furthermore, we can write $x_{n}^{\prime}=g_{n}^{\prime}+b_{n}^{\prime}$, were $g_{n}^{\prime}=g_{n}+\chi_{\omega_{n}} z$ and $b_{n}^{\prime}=b_{n}+\chi_{\mathbf{T} \backslash \omega_{n}} z$. Condition (b1) is verified by the definition of $\omega_{n}$, and because $\|z(\zeta)\| \leq \frac{1}{2}$ almost everywhere. To verify (b2) we calculate

$$
\begin{aligned}
& \left\|b_{n}^{\prime}\right\|^{2}-\eta^{2}\left\|g_{n}^{\prime}\right\|^{2} \\
& \quad=\left\|b_{n}\right\|^{2}-\eta^{2}\left\|g_{n}\right\|^{2}+\left\|\chi_{\mathbf{T} \backslash \omega_{n}} z\right\|^{2}-\eta^{2}\left\|\chi_{\omega_{n}} z\right\|^{2} \\
& \quad+2 \operatorname{Re}\left[\left\langle b_{n}, \chi_{\mathbf{T} \backslash \omega_{n}} z\right\rangle-\eta^{2}\left\langle g_{n}, \chi_{\omega_{n}} z\right\rangle\right] \\
& \quad \leq\left\|\chi_{\mathbf{T} \backslash \omega_{n}} z\right\|^{2}-\eta^{2}\left\|\chi_{\omega_{n}} z\right\|^{2}+2 \operatorname{Re}\left[\left\langle\chi_{\mathbf{T} \backslash \omega_{n}} b_{n}, z\right\rangle-\eta^{2}\left\langle\chi_{\omega_{n}} g_{n}, z\right\rangle\right],
\end{aligned}
$$

where we used the fact that $x_{n}$ satisfies (b2). We use now (ii), (iii), (v) and (vi) to get

$$
\begin{aligned}
& \limsup _{n \rightarrow \infty}\left(\left\|b_{n}^{\prime}\right\|^{2}-\eta^{2}\left\|g_{n}^{\prime}\right\|^{2}\right) \\
& \quad \leq\left\|(1-f)^{1 / 2} z\right\|^{2}-\eta^{2}\left\|f^{1 / 2} z\right\|^{2}+2 \operatorname{Re}\left[\langle v, z\rangle-\eta^{2}\langle u, z\rangle\right] \\
& \quad=\left\|(1-f)^{1 / 2} z\right\|^{2}-\eta^{2}\left\|f^{1 / 2} z\right\|^{2}<0,
\end{aligned}
$$


so that $x_{n}^{\prime}$ satisfies (b2) eventually. Finally,

$$
\begin{aligned}
\left|\left\langle g_{n}^{\prime}, \xi_{j}\right\rangle\right| & \leq\left|\left\langle g_{n}, \xi_{j}\right\rangle\right|+\left|\left\langle\chi_{\omega_{n}} z, \xi_{j}\right\rangle\right| \\
& \leq \delta\left\|x_{n}\right\|+\left|\left\langle\chi_{\omega_{n}} z, \xi_{j}\right\rangle\right| \\
& \leq \delta \gamma|\sigma|^{1 / 2}+\left|\left\langle\chi_{\omega_{n}} z, \xi_{j}\right\rangle\right|
\end{aligned}
$$

so that using (iv) and (v) we get

$$
\limsup _{n \rightarrow \infty}\left|\left\langle g_{n}^{\prime}, \xi_{j}\right\rangle\right| \leq \delta \gamma|\sigma|^{1 / 2}+\left|\left\langle f z, \xi_{j}\right\rangle\right|=\delta \gamma|\sigma|^{1 / 2},
$$

and hence $\left|\left\langle g_{n}^{\prime}, \xi_{j}\right\rangle\right|<\delta\left\|x_{n}^{\prime}\right\|$ for $n$ large. Therefore $x_{n}^{\prime} \in S$ eventually, and this is a contradiction because $\left\|x_{n}^{\prime}\right\|>\sup \{\|x\|: x \in S\}$ for $n$ large. Q.E.D.

We are now able to prove a strengthening of Proposition 6.2.

\subsection{Proposition. If $\eta<\frac{1}{2}$ then}

$$
\sup \left\{\|x\|: x \in S\left(\sigma ; \xi_{1}, \xi_{2}, \ldots, \xi_{p} ; \eta ; \delta\right)\right\} \geq(1-\eta)|\sigma|^{1 / 2} .
$$

Proof. Denote, as before, $S=S\left(\sigma ; \xi_{1}, \xi_{2}, \ldots, \xi_{p} ; \eta ; \delta\right)$, and set

$$
\sup \{\|x\|: x \in S\}=\mu(1-\eta)|\sigma|^{1 / 2} .
$$

Suppose, to get a contradiction, that $\mu<1$. Let $x=g+b$ be an element of $S$, and note that $\|g\| \leq \mu|\sigma|^{1 / 2}$. The set $\omega=\{\zeta \in$ $\left.\sigma:\|g(\zeta)\|<\mu^{1 / 2}\right\}$ has positive measure; in fact

$$
|\sigma \backslash \omega|=\frac{1}{2 \pi} \int_{\sigma \backslash \omega} d \theta \leq \frac{1}{\mu} \frac{1}{2 \pi} \int_{\sigma \backslash \omega}\left\|g\left(e^{i \theta}\right)\right\|^{2} d \theta \leq \mu|\sigma|,
$$

so that $|\omega| \geq(1-\mu)|\sigma|$. Let now $\delta^{\prime}$ be a positive number, and choose an element

$$
z=\gamma+\beta \in S^{\prime}=S\left(\omega ; b, g, \xi_{1}, \xi_{2}, \ldots, \xi_{p} ; \eta ; \delta^{\prime}\right)
$$

such that $\|z\| \geq 2^{-3} \eta|\omega|^{1 / 2} \geq 2^{-3} \eta(1-\mu)^{1 / 2}|\sigma|^{1 / 2}$. Let us assume for the moment that the vector $x^{\prime}=x+\left(1-\mu^{1 / 2}\right) z$ has been shown to belong to $S$. We must have then $\left\|x^{\prime}\right\| \leq \mu(1-$ $\eta)|\sigma|^{1 / 2}$ or, equivalently since $\langle x, z\rangle=0,\|x\|^{2}+\left(1-\mu^{1 / 2}\right)^{2}\|z\|^{2} \leq$ $\mu^{2}(1-\eta)^{2}|\sigma|$. Thus

$$
\|x\|^{2}+\left(1-\mu^{1 / 2}\right)^{2} 2^{-6} \eta^{2}(1-\mu)|\sigma| \leq \mu^{2}(1-\eta)^{2}|\sigma| .
$$

Letting $\|x\|$ approach $\mu(1-\eta)|\sigma|$ we get $\left(1-\mu^{1 / 2}\right)^{2} 2^{-6} \eta^{2}(1-\eta)|\sigma|$ $\leq 0$, a contradiction. Thus, to conclude the proof it suffices to show that $x^{\prime} \in S$ for sufficiently small $\delta^{\prime}$. To do this note that condition (a) of Definition 6.1 is clearly verified. Next we write $x^{\prime}=g^{\prime}+b^{\prime}$, were $g^{\prime}=g+\left(1-\mu^{1 / 2}\right) \gamma$ and $b^{\prime}=b+\left(1-\mu^{1 / 2}\right) \beta$. 
We have

$$
\begin{aligned}
\left\|g^{\prime}(\zeta)\right\| & \leq\|g(\zeta)\|+\left(1-\mu^{1 / 2}\right)\|\gamma(\zeta)\| \\
& \leq\|g(\zeta)\|+\left(1-\mu^{1 / 2}\right) \chi_{\omega}(\zeta) \\
& \leq \chi_{\sigma}(\zeta)
\end{aligned}
$$

by the definition of the set $\omega$; this shows that (b1) of Definition 6.1 holds for $x^{\prime}$. To verify (b2) we calculate

$$
\begin{aligned}
\left\|b^{\prime}\right\|^{2}-\eta^{2}\left\|g^{\prime}\right\|^{2}= & \|b\|^{2}-\eta^{2}\|g\|^{2}+\left(1-\mu^{1 / 2}\right)^{2}\left(\|\beta\|^{2}-\eta^{2}\|\gamma\|^{2}\right) \\
& +2\left(1-\mu^{1 / 2}\right) \operatorname{Re}\left[\langle g, \gamma\rangle-\eta^{2}\langle b, \beta\rangle\right] \\
\leq & \|b\|^{2}-\eta^{2}\|g\|^{2}+2[|\langle g, \gamma\rangle|+|\langle b, \beta\rangle|] .
\end{aligned}
$$

Using the fact that $z \in S^{\prime}$ we get $|\langle g, \gamma\rangle| \leq \delta^{\prime}\|z\| \leq 2 \delta^{\prime}$ and $|\langle b, \beta\rangle|=|\langle b, \gamma\rangle| \leq \delta^{\prime}\|z\| \leq 2 \delta^{\prime}$, so that

$$
\left\|b^{\prime}\right\|^{2}-\eta^{2}\left\|g^{\prime}\right\|^{2} \leq\|b\|^{2}-\eta^{2}\|g\|^{2}+8 \delta^{\prime} .
$$

Therefore (b2) is verified provided that

$$
8 \delta^{\prime}<\eta^{2}\|g\|^{2}-\|b\|^{2} \text {. }
$$

To verify (b3) we use the fact that $x \in S$ and $z \in S^{\prime}$ to get

$$
\begin{aligned}
\left|\left\langle x^{\prime}, \xi_{j}\right\rangle\right| & \leq\left|\left\langle x, \xi_{j}\right\rangle\right|+\left(1-\mu^{1 / 2}\right)\left|\left\langle z, \xi_{j}\right\rangle\right| \\
& \leq\left|\left\langle x, \xi_{j}\right\rangle\right|+\left|\left\langle z, \xi_{j}\right\rangle\right| \\
& \leq \delta\|x\|+\delta^{\prime}\|z\| \\
& \leq \delta\|x\|+2 \delta^{\prime} .
\end{aligned}
$$

Since

$$
\begin{aligned}
\left\|x^{\prime}\right\| & =\left(\|x\|^{2}+\left(1-\mu^{1 / 2}\right)^{2}\|z\|^{2}\right)^{1 / 2} \\
& \geq\left[\|x\|^{2}+\left(1-\mu^{1 / 2}\right)^{2} 2^{-6} \eta^{2}(1-\mu)|\sigma|\right]^{1 / 2},
\end{aligned}
$$

we see that $(6.3)$ is satisfied provided that

$$
\delta\|x\|+2 \delta^{\prime}<\delta\left[\|x\|^{2}+\left(1-\mu^{1 / 2}\right)^{2} 2^{-6} \eta^{2}(1-\mu)|\sigma|\right]^{1 / 2} .
$$

Since both (6.4) and (6.5) are satisfied for sufficiently small $\delta^{\prime}$, the proposition follows. Q.E.D.

The left-hand side in the inequality of Proposition 4.3 is increasing in $\eta$, while the right-hand side is decreasing. Thus Proposition 4.3 immediately implies the following strengthening of itself. 
6.6. Corollary. For all $\eta>0$ we have

$$
\sup \left\{\|x\|: x \in S\left(\sigma ; \xi_{1}, \xi_{2}, \ldots, \xi_{p} ; \eta ; \delta\right\} \geq|\sigma|^{1 / 2} .\right.
$$

\section{APPROXIMATE FACTORIZATION IN $L^{1}$}

The following result is a little weaker than Theorem 5.1 because it provides approximate factorization, and a little stronger than Theorem 5.1 because it refers to functions in $L^{1}$ rather than classes in $L^{1} / H_{0}^{1}$. The notation and assumptions of the previous section will remain in force.

7.1. Theorem. Let $\xi_{1}, \xi_{2}, \ldots, \xi_{p} \in \mathscr{H}, \varepsilon>0$, and $f \in L^{1}$ be given. There exist $x, y \in \mathscr{H}$ such that $\|x\| \leq\|f\|_{1}^{1 / 2}$, $\|y\| \leq\|f\|_{1}^{1 / 2},\left\langle x, \xi_{j}\right\rangle=\left\langle y, \xi_{j}\right\rangle=0$ for $j=1,2, \ldots, p$, and $\|f-x \cdot y\|_{1}<\varepsilon$. If $f \geq 0$ almost everywhere we may choose $x=y$.

Proof. Choose pairwise disjoint sets $\sigma_{1}, \sigma_{2}, \ldots, \sigma_{n}$ with positive measure, and scalars $\gamma_{1}, \gamma_{2}, \ldots, \gamma_{n}$ such that

$$
\left\|f-\sum_{i=1}^{n} \gamma_{i} \chi_{\sigma_{i}}\right\|_{1}<\frac{\varepsilon}{2},
$$

and

$$
\sum_{i=1}^{n}\left|\gamma_{i}\right|\left|\sigma_{i}\right|<\|f\|_{1}
$$

Fix $\eta, \delta>0$, and choose by virtue of Corollary 6.6 vectors

$$
z_{i} \in S\left(\sigma_{i} ; \xi_{1}, \xi_{2}, \ldots, \xi_{p} ; \eta ; \delta\right)
$$

such that

$$
\left\|z_{i}\right\| \geq(1-\eta)\left|\sigma_{i}\right|^{1 / 2}, \quad i=1,2, \ldots, n .
$$

Choose for each $i$ a square root $\alpha_{i}$ of $\gamma_{i}$, and set

$$
x=\sum_{i=1}^{n} \alpha_{i} z_{i}, \quad y=\sum_{i=1}^{n} \overline{\alpha_{i}} z_{i} .
$$

If $f$ is positive then the $\gamma_{i}$ can be assumed positive, and hence $x=y$. To conclude the proof we have to show that $x$ and $y$ satisfy the requirements of the statement provided that $\eta$ is appropriately small. The conditions $\left\langle x, \xi_{j}\right\rangle=\left\langle y, \xi_{j}\right\rangle=0,1 \leq j \leq p$, are immediate. Write $z_{i}=g_{i}+b_{i}$ as required by the fact that 
$z_{i} \in S\left(\sigma_{i} ; \xi_{1}, \xi_{2}, \ldots, \xi_{p} ; \eta ; \delta\right)$, and note that the $g_{i}$ have disjoint supports, hence they are pairwise orthogonal. Therefore

$$
\begin{aligned}
\|x\|^{2} & \leq \sum_{i=1}^{n}\left|\gamma_{i}\right|\left|\sigma_{i}\right|+\sum_{i, j=1}^{n}\left|\alpha_{i} \| \alpha_{j}\right|\left(2\left\|g_{i}\right\|\left\|b_{j}\right\|+\left\|b_{i}\right\|\left\|b_{j}\right\|\right) \\
& \leq \sum_{i=1}^{n}\left|\gamma_{i}\right|\left|\sigma_{i}\right|+\sum_{i, j=1}^{n}\left|\alpha_{i} \| \alpha_{j}\right|\left(2 \eta\left\|g_{i}\right\|\left\|g_{j}\right\|+\eta^{2}\left\|g_{i}\right\|\left\|g_{j}\right\|\right) \\
& \leq \sum_{i=1}^{n}\left|\gamma_{i} \| \sigma_{i}\right|+\left(2 \eta+\eta^{2}\right)\left(\sum_{i=1}^{n}\left|\alpha_{i}\right|\right)^{2}
\end{aligned}
$$

and by (7.3) we see that $\|x\| \leq\|f\|_{1}^{1 / 2}$ if $\eta$ is small enough. Finally we note that

$$
x \cdot y=\sum_{i=1}^{n} \gamma_{i} g_{i} \cdot g_{i}+\sum_{i, j=1}^{n} \alpha_{i} \alpha_{j}\left(g_{i} \cdot b_{j}+b_{i} \cdot g_{j}+b_{i} \cdot b_{j}\right),
$$

where we used the fact that $g_{i} \cdot g_{j}=0$ if $i \neq j$. Furthermore,

$$
\begin{aligned}
\left\|\chi_{\sigma_{i}}-g_{i} \cdot g_{i}\right\|_{1} & =\frac{1}{2 \pi} \int_{0}^{2 \pi}\left(\chi_{\sigma_{i}}\left(e^{i \theta}\right)-\left\|g_{i}\left(e^{i \theta}\right)\right\|^{2}\right) d \theta \\
& =\left|\sigma_{i}\right|-\left\|g_{i}\right\|^{2} \\
& \leq\left|\sigma_{i}\right|-\frac{\left\|x_{i}\right\|^{2}}{(1+\eta)^{2}} \\
& \leq\left|\sigma_{i}\right|-\left(\frac{1-\eta}{1+\eta}\right)^{2}\left|\sigma_{i}\right|,
\end{aligned}
$$

whence

$$
\begin{aligned}
& \left\|\sum_{i=1}^{n} \gamma_{i} \chi_{\sigma_{i}}-x \cdot y\right\|_{1} \leq \sum_{i=1}^{n}\left(1-\left(\frac{1-\eta}{1+\eta}\right)^{2}\right)\left|\gamma_{i} \| \sigma_{i}\right| \\
& \quad+\sum_{i, j=1}^{n}\left|\alpha_{i} \| \alpha_{j}\right|\left[\left\|g_{i}\right\|\left\|b_{j}\right\|+\left\|b_{i}\right\|\left\|g_{j}\right\|+\left\|b_{i}\right\|\left\|b_{j}\right\|\right] \\
& \quad \leq\left(1-\left(\frac{1-\eta}{1+\eta}\right)^{2}\right) \sum_{i=1}^{n}\left|\gamma_{i}\right|\left|\sigma_{i}\right|+\left(2 \eta+\eta^{2}\right)\left(\sum_{i=1}^{n}\left|\alpha_{i}\right|\right)^{2} .
\end{aligned}
$$

This last number can be made $<\varepsilon / 2$ if $\eta$ is sufficiently small, and the inequality $\|f-x \cdot y\|<\varepsilon$ follows from (7.2). Q.E.D.

\section{FACTORIZATION IN THE QUOTIENT SPACE}

In this section we finally prove Theorem 5.1 and conclude the paper. The ideas here can be traced back to [12], but they have 
been adapted to the case of contractions of class $C_{0}$. We will need to return to our contraction $T$, subject to Assumptions 2.3, 2.5 and 2.8. Let

$$
\mathscr{K}_{+}=\bigvee_{n=0}^{\infty} U^{n} \mathscr{H} \quad \text { and } \quad U_{+}=U \mid \mathscr{K}_{+} .
$$

8.1. Lemma. If $x \in \mathscr{K}_{+}$and $y \in \mathscr{H}$ then $[x \cdot y]=\left[P_{\mathscr{H}} x \cdot y\right]$. Proof. We note first that $\mathscr{K}_{+} \ominus \mathscr{H}$ is invariant for $U_{+}$. Indeed, we have

$$
\mathscr{K}_{+}=\mathscr{H} \bigvee\left\{U^{n} h-T^{n} h: n \geq 1, h \in \mathscr{H}\right\},
$$

and $\mathscr{H} \perp\left\{U^{n} h-T^{n} h: n \geq 1, h \in \mathscr{H}\right\}$ because $U$ is a dilation of $T$. Thus we have

$$
\mathscr{H}_{+} \ominus \mathscr{H}=\bigvee\left\{U^{n} h-T^{n} h: n \geq 1, h \in \mathscr{H}\right\}
$$

Since

$$
U_{+}\left(U^{n} h-T^{n} h\right)=U^{n+1} h-T^{n+1} h+U k-T k, \quad k=-T^{n} h,
$$

we conclude immediately that $U_{+}$leaves $\mathscr{K}_{+} \ominus \mathscr{H}$ invariant. This, of course amounts to the identity $P_{\mathscr{H}} U_{+}^{n} P_{\mathscr{H}}=P_{\mathscr{H}} U_{+}^{n}, n \geq 1$. Thus for $x \in \mathscr{K}_{+}$and $y \in \mathscr{H}$ we have

$$
\left\langle U_{+}^{n} x, y\right\rangle=\left\langle P_{\mathscr{H}} U_{+}^{n} x, y\right\rangle=\left\langle P_{\mathscr{H}} U_{+}^{n} P_{\mathscr{H}} x, y\right\rangle=\left\langle U_{+}^{n} P_{\mathscr{H}} x, y\right\rangle
$$

for all $n \geq 0$. Q.E.D.

We note for further use the relation $T^{*}=U_{+}^{*} \mid \mathscr{H}$ which follows from the fact that $\mathscr{K}_{+} \ominus \mathscr{H}$ is invariant for $U_{+}$.

8.2. Proposition. If $x \in \mathscr{K}_{+}$and $\left\{y_{n}\right\} \subset \mathscr{H}$ is a sequence that converges weakly to zero, then $\lim _{n \rightarrow \infty}\left\|\left[x^{\prime} \cdot y_{n}\right]\right\|=0$.

Proof. By Lemma 8.1, we may assume that $x \in \mathscr{H}$, and by Proposition 4.5(i) it will suffice to prove the present result for all vectors $x \in \bigoplus_{n=1}^{\infty} U^{* n} \mathscr{V}$. Since $\left\{y_{n}\right\}$ is a bounded sequence, it suffices to prove the proposition for $x$ in a total set in $\bigoplus_{n=1}^{\infty} U^{* n} \mathscr{V}$, for instance for $x$ of the form $U^{* k} z, z \in \mathscr{V}, k \geq 1$. Note that $\left\langle U^{p} x, y_{n}\right\rangle=0$ if $p \geq k$, and therefore

$$
\left\|\left[x \cdot y_{n}\right]\right\| \leq \sum_{p=0}^{k-1}\left|\left\langle U^{p} x, y_{n}\right\rangle\right| .
$$

The latter sum clearly converges to zero as $n \rightarrow \infty$. Q.E.D. 
We use now the fact that $U_{+}$is an isometry to write $\mathscr{K}_{+}=$ $\mathscr{M} \oplus \mathscr{R}$, where $\mathscr{M}$ and $\mathscr{R}$ are reducing spaces for $U_{+}, U_{+} \mid \mathscr{M}^{+}$is a unilateral shift (pure isometry), and $U_{+} \mid \mathscr{R}$ is a unitary operator. If $m, m^{\prime} \in \mathscr{M}$ and $r, r^{\prime} \in \mathscr{R}$ it is clear that

$$
(m+r) \cdot\left(m^{\prime}+r^{\prime}\right)=m \cdot m^{\prime}+r \cdot r^{\prime}
$$

(just check the Fourier coefficients).

8.3. Proposition. If $y \in \mathscr{K}$ and $\left\{x_{n}\right\} \subset \mathscr{M}$ is a sequence that converges weakly to zero, then $\lim _{n \rightarrow \infty}\left\|\left[x_{n} \cdot y\right]\right\|=0$.

Proof. This is similar to Proposition 8.2. It suffices, by the remark preceding the statement, to prove the proposition for a set of vectors $y$ total in $\mathscr{M}$. Assume therefore that $y \in \operatorname{ker} U_{+}^{* N}$ for some $N \geq 1$. Then $\left\langle U_{+}^{k} x_{n}, y\right\rangle=0$ for $k \geq N$, and hence

$$
\left\|\left[x_{n} \cdot y\right]\right\| \leq \sum_{k=0}^{N-1}\left|\left\langle U_{+}^{k} x_{n}, y\right\rangle\right| \text {. Q.E.D. }
$$

We proceed now to several successive improvements of Theorem 7.1 .

8.4. Proposition. Let $\varepsilon>0, x, y, y^{\prime} \in \mathscr{H}$, and $x^{\prime} \in \mathscr{R}$ be given. There exist $x_{1}, y_{1} \in \mathscr{H}$ with the following properties

$$
\begin{gathered}
\left\|[x \cdot y]+\left[x^{\prime} \cdot y^{\prime}\right]-\left[x_{1} \cdot y_{1}\right]\right\|<\varepsilon, \\
\left\|y-y_{1}\right\| \leq 2\left\|y^{\prime}\right\| \text { and } \\
\left\|x_{1}\right\| \leq(1+\varepsilon)\left[\left\|P_{\mathscr{M}} x\right\|^{2}+\left(\left\|P_{\mathscr{R}} x\right\|+\left\|x^{\prime}\right\|\right)^{2}\right]^{1 / 2}
\end{gathered}
$$

Proof. To explain the idea, we note that we may not be able to write

$$
[\dot{x} \cdot y]+\left[x^{\prime} \cdot y^{\prime}\right]=\left[x_{1} \cdot\left(y+y^{\prime}\right)\right]
$$

for some $x_{1} \in \mathscr{H}$ because the sum $y+y^{\prime}$ may vanish on a large set. Therefore we have to adjust the vector $y^{\prime}$ such that the sum $y+y^{\prime}$ is not zero two often. The adjustment of $y^{\prime}$ is done as follows. Fix $\delta>0$, define a measurable set $\sigma \subset \mathbf{T}$ by

$$
\sigma=\left\{\zeta \in \mathbf{T}:\left\|\left(P_{\mathscr{R}} y^{\prime}\right)(\zeta)\right\| \leq\left\|\left(P_{\mathscr{R}} y\right)(\zeta)\right\|\right\},
$$

and consider an outer function $\psi \in H^{\infty}$ such that $|\psi(\zeta)|=\delta$ almost

everywhere on $\sigma$ and $|\psi(\zeta)|=2$ almost everywhere on $\mathbf{T} \backslash \sigma$. Define $\eta \in \mathscr{H}$ by $\eta=\psi(T)^{*} y^{\prime}$, and note that $\psi(T)^{*} y^{\prime}=\psi\left(U_{+}^{*}\right) y^{\prime}$, and $\psi\left(U_{+}^{*}\right) \mid \mathscr{R}$ is the operator of pointwise multiplication by $\bar{\psi}$. 
Therefore $\left\|\left(P_{\mathscr{R}} \eta\right)(\zeta)\right\|=|\psi(\zeta)|\left\|\left(P_{\mathscr{R}} y^{\prime}\right)(\zeta)\right\|$ for almost every $\zeta$. We need to make one further change. Choose a natural number $N$ such that $\left\|U_{+}^{* N} P_{\mathscr{M}} \eta\right\|<\delta$ and set $y^{\prime \prime}=T^{* N} \eta \in \mathscr{H}$. We have then

$\left\|P_{\mathscr{M}} y^{\prime \prime}\right\|=\left\|P_{\mathscr{M}} T^{* n} \eta\right\|=\left\|P_{\mathscr{M}} U_{+}^{* n} \eta\right\|<\delta, \quad\left\|y^{\prime \prime}\right\| \leq\|\eta\| \leq 2\left\|y^{\prime}\right\|$, and

$$
\left\|\left(P_{\mathscr{R}} y\right)(\zeta)\right\|=\left\|\left(P_{\mathscr{R}} \eta\right)(\zeta)\right\|=|\psi(\zeta)|\left\|\left(P_{\mathscr{R}} y^{\prime}\right)(\zeta)\right\|
$$

almost everywhere. We are now ready to define $y_{1}=y+y^{\prime \prime}$. In order to define $x_{1}$ we note first that for almost every $\zeta \in \sigma$,

$$
\begin{aligned}
\left\|\left(P_{\mathscr{R}} y_{1}\right)(\zeta)\right\| & =\left\|\left(P_{\mathscr{R}} y\right)(\zeta)+\left(P_{\mathscr{R}} y^{\prime \prime}\right)(\zeta)\right\| \\
& \geq\left\|\left(P_{\mathscr{R}} y\right)(\zeta)\right\|-\left\|\left(P_{\mathscr{R}} y^{\prime \prime}\right)(\zeta)\right\| \\
& =\left\|\left(P_{\mathscr{R}} y\right)(\zeta)\right\|-\delta\left\|\left(P_{\mathscr{R}} y^{\prime}\right)(\zeta)\right\| \\
& \geq(1-\delta)\left\|\left(P_{\mathscr{R}} y\right)(\zeta)\right\|,
\end{aligned}
$$

while for almost every $\zeta \in \mathbf{T} \backslash \sigma$,

$$
\begin{aligned}
\left\|\left(P_{\mathscr{R}} y_{1}\right)(\zeta)\right\| & \geq\left\|\left(P_{\mathscr{R}} y^{\prime \prime}\right)(\zeta)\right\|-\left\|\left(P_{\mathscr{R}} y\right)(\zeta)\right\| \\
& =2\left\|\left(P_{\mathscr{R}} y^{\prime}\right)(\zeta)\right\|-\left\|\left(P_{\mathscr{R}} y\right)(\zeta)\right\| \\
& \geq\left\|\left(P_{\mathscr{R}} y^{\prime}\right)(\zeta)\right\| .
\end{aligned}
$$

Therefore we have

$$
\left\|\left(P_{\mathscr{R}} y_{1}\right)(\zeta)\right\| \geq(1-\delta) \max \left\{\left\|\left(P_{\mathscr{R}} y\right)(\zeta)\right\|,\left\|\left(P_{\mathscr{R}} y^{\prime}\right)(\zeta)\right\|\right\}
$$

almost everywhere. It follows that we can choose a measurable function $g(\zeta)$ such that

$$
g(\zeta)\left\|\left(P_{\mathscr{R}} y_{1}\right)(\zeta)\right\|^{2}=\left(x \cdot P_{\mathscr{R}} y\right)(\zeta)+\left(x^{\prime} \cdot y^{\prime}\right)(\zeta)
$$

almost everywhere, and $g(\zeta)=0$ when $\left(P_{\mathscr{R}} y_{1}\right)(\zeta)=0$. Moreover, we have

$$
\begin{aligned}
g(\zeta)\left\|\left(P_{\mathscr{R}} y_{1}\right)(\zeta)\right\| \\
\quad \leq \frac{\left\|\left(P_{\mathscr{R}} x\right)(\zeta)\right\|\left\|\left(P_{\mathscr{R}} y\right)(\zeta)\right\|+\left\|x^{\prime}(\zeta)\right\|\left\|\left(P_{\mathscr{R}} y^{\prime}\right)(\zeta)\right\|}{\left\|\left(P_{\mathscr{R}} y_{1}\right)(\zeta)\right\|} \\
\quad \leq \frac{\left(\left\|\left(P_{\mathscr{R}} x\right)(\zeta)\right\|+\left\|x^{\prime}(\zeta)\right\|\right) \max \left\{\left\|\left(P_{\mathscr{R}} y\right)(\zeta)\right\|,\left\|\left(P_{\mathscr{R}} y^{\prime}\right)(\zeta)\right\|\right\}}{\left\|\left(P_{\mathscr{R}} y_{1}\right)(\zeta)\right\|} \\
\quad \leq \frac{1}{1-\delta}\left(\left\|\left(P_{\mathscr{R}} x\right)(\zeta)\right\|+\left\|x^{\prime}(\zeta)\right\|\right)
\end{aligned}
$$

almost everywhere. Therefore the function $x_{2} \in \mathscr{R}$ defined by $x_{2}(\zeta)=g(\zeta)\left(P_{\mathscr{R}} y_{1}\right)(\zeta)$ satisfies the inequality

$\left\|x_{2}\right\| \leq \frac{1}{1-\delta}\left(\left\|P_{\mathscr{R}} x\right\|+\left\|x^{\prime}\right\|\right), \quad$ and $\quad x_{2} \cdot y_{1}=x \cdot\left(P_{\mathscr{R}} y\right)+x^{\prime} \cdot y^{\prime}$. 
Define next $x_{1}=P_{\mathscr{H}}\left(P_{\mathscr{M}} x+x_{2}\right)$. To conclude the proof we have to show that $x_{1}$ and $y_{1}$ satisfy the conditions in the statement provided that $\delta$ is sufficiently small. First,

$$
\left\|y-y_{1}\right\|=\left\|y^{\prime \prime}\right\| \leq 2\left\|y^{\prime}\right\| \text {, }
$$

and

$$
\begin{aligned}
\left\|x_{1}\right\| & \leq\left\|P_{\mathscr{M}} x+x_{2}\right\|=\left[\left\|P_{\mathscr{M}} x\right\|^{2}+\left\|x_{2}\right\|^{2}\right]^{1 / 2} \\
& \leq\left[\left\|P_{\mathscr{M}} x\right\|^{2}+\frac{1}{(1-\delta)^{2}}\left(\left\|P_{\mathscr{R}} x\right\|+\left\|x^{\prime}\right\|\right)^{2}\right]^{1 / 2} \\
& \leq \frac{1}{1-\delta}\left[\left\|P_{\mathscr{M}} x\right\|^{2}+\left(\left\|P_{\mathscr{R}} x\right\|+\left\|x^{\prime}\right\|\right)^{2}\right]^{1 / 2},
\end{aligned}
$$

so that the last inequality in the lemma is satisfied if $1 /(1-\delta)<$ $1+\varepsilon$. Finally, we have $\left[x_{1} \cdot y_{1}\right]=\left[\left(P_{\mathscr{M}} x+x_{2}\right) \cdot y_{1}\right]$ by Lemma 8.1, and therefore

$$
\begin{aligned}
{[x \cdot y] } & +\left[x^{\prime} \cdot y^{\prime}\right]-\left[x_{1} \cdot y_{1}\right] \\
= & {\left[P_{\mathscr{M}} x \cdot y\right]+\left[x \cdot P_{\mathscr{R}} y\right]+\left[x^{\prime} \cdot y^{\prime}\right]-\left[P_{\mathscr{M}} x \cdot y\right] } \\
& -\left[P_{\mathscr{M}} x \cdot P_{\mathscr{M}} y^{\prime \prime}\right]-\left[x_{2} \cdot P_{\mathscr{R}} y_{1}\right] \\
= & -\left[P_{\mathscr{M}} x \cdot P_{\mathscr{M}} y^{\prime \prime}\right] .
\end{aligned}
$$

Thus

$$
\left\|[x \cdot y]+\left[x^{\prime} \cdot y^{\prime}\right]-\left[x_{1} \cdot y_{1}\right]\right\| \leq\|x\|\left\|P_{\mathscr{M}} y^{\prime \prime}\right\| \leq \delta\|x\|<\varepsilon
$$

for $\delta$ sufficiently small. Q.E.D.

Our next task is to replace the function $x^{\prime} \cdot y^{\prime}$ in Proposition 8.4 by an arbitrary function in $L^{1}$.

8.5. Proposition. Let $\varepsilon>0, x, y \in \mathscr{H}$, and $g \in L^{1}$ be given. There exist $x_{1}, y_{1} \in \mathscr{H}$ with the following properties

$$
\begin{aligned}
& \left\|[x \cdot y]+[g]-\left[x_{1} \cdot y_{1}\right]\right\|<\varepsilon, \quad\left\|y-y_{1}\right\| \leq 3\|g\|_{1}^{1 / 2}, \text { and } \\
& \left\|x_{1}\right\| \leq(1+\varepsilon)\left[\left(\left\|P_{\mathscr{M}} x\right\|+\|g\|_{1}^{1 / 2}\right)^{2}+\left(\left\|P_{\mathscr{R}} x\right\|+\|g\|_{1}^{1 / 2}\right)^{2}\right]^{1 / 2} .
\end{aligned}
$$

Proof. Theorem 7.1 implies the existence of orthogonal sequences $\left\{x^{(n)}\right\},\left\{y^{(n)}\right\}$ in $\mathscr{H}$ such that $\left\|x^{(n)}\right\| \leq\|g\|_{1}^{1 / 2},\left\|y^{(n)}\right\| \leq\|g\|_{1}^{1 / 2}$, and

$$
\lim _{n \rightarrow \infty}\left\|g-x^{(n)} \cdot y^{(n)}\right\|_{1}=0 .
$$

Fix $\delta>0, \delta<\varepsilon / 4$, and note that Propositions 8.2 and 8.3 imply the existence of $n$ such that, upon setting $\xi=x^{(n)}$ and $\eta=y^{(n)}$, 
we have $\|[x \cdot \eta]\|<\delta,\left\|\left[\left(P_{\mathscr{M}} \xi\right) \cdot y\right]\right\|<\delta$, and $\|g-\xi \cdot \eta\|_{1}<\delta$. Observe that

$$
\begin{aligned}
{[x \cdot y]+[g]=} & {[x \cdot y]+[\xi \cdot \eta]+[g-\xi \cdot \eta] } \\
= & {[x \cdot y]+\left[P_{\mathscr{M}} \xi \cdot \eta\right]+\left[P_{\mathscr{R}} \xi \cdot \eta\right]+[g-\xi \cdot \eta] } \\
= & {\left[\left(x+P_{\mathscr{M}} \xi\right) \cdot(y+\eta)\right]+\left[P_{\mathscr{R}} \xi \cdot \eta\right]+[g-\xi \cdot \eta] } \\
& -[x \cdot \eta]-\left[P_{\mathscr{M}} \xi \cdot y\right],
\end{aligned}
$$

or, using Lemma 8.1,

$$
[x \cdot y]+[g]=\left[P_{\mathscr{H}}\left(x+P_{\mathscr{M}} \xi\right) \cdot(y+\eta)\right]+\left[P_{\mathscr{R}} \xi \cdot \eta\right]+r,
$$

where the remainder $r$ has norm $\|r\|<3 \delta$. We can now apply Proposition 8.4, with $\varepsilon, x, y, x^{\prime}, y^{\prime}$ of that proposition replaced by $\delta, P_{\mathscr{P}}\left(x+P_{\mathscr{M}} \xi\right), y+\eta, P_{\mathscr{R}} \xi, \eta$, respectively. This yields vectors $x_{1}$ and $y_{1}$ with the following properties

$$
\begin{gathered}
\left\|\left[P_{\mathscr{H}}\left(x+P_{\mathscr{M}} \xi\right) \cdot(y+\eta)\right]+\left[P_{\mathscr{R}} \xi \cdot \eta\right]-\left[x_{1} \cdot y_{1}\right]\right\|<\delta, \\
\left\|y+\eta-y_{1}\right\| \leq 2\|\eta\| \leq 2\|g\|_{1}^{1 / 2},
\end{gathered}
$$

and

$$
\left\|x_{1}\right\| \leq(1+\delta)\left[\left\|P_{\mathscr{M}} x+P_{\mathscr{M}} \xi\right\|^{2}+\left(\left\|P_{\mathscr{R}} x\right\|+\left\|P_{\mathscr{R}} \xi\right\|\right)^{2}\right]^{1 / 2} .
$$

It is immediate now to check that $x_{1}$ and $y_{1}$ verify the conclusion of our proposition. Q.E.D.

The following result is a slightly modified form of Theorem 5.1.

8.6. Theorem. Given $f \in L^{1}$ and $\varepsilon>0$ there exist $x, y \in \mathscr{H}$ such that $[f]=[x \cdot y]$ and $\|x\| \leq(1+\varepsilon)\|f\|_{1}^{1 / 2},\|y\| \leq(1+\varepsilon)$ $\times\|f\|_{1}^{1 / 2}$.

Proof. We may, and shall, assume without loss of generality that $f \neq 0$. Let $\left\{\delta_{n}\right\}$ be a sequence of positive numbers, subject to several conditions to be specified shortly. We claim that there exist sequences $\left\{x_{n}\right\}$ and $\left\{y_{n}\right\}$ in $\mathscr{H}$ with the following properties:

$$
\begin{gathered}
\left\|[f]-\left[x_{n} \cdot y_{n}\right]\right\|<\delta_{n}^{2} \\
\left\|x_{0}\right\| \leq\|f\|_{1}^{1 / 2},\left\|y_{0}\right\| \leq\|f\|_{1}^{1 / 2}, \quad\left\|y_{n+1}-y_{n}\right\| \leq 3 \delta_{n}, \text { and } \\
\left\|x_{n+1}\right\| \leq\left(1+\delta_{n+1}\right)\left[\left(\left\|P_{\mathscr{M}} x_{n}\right\|+\delta_{n}\right)^{2}+\left(\left\|P_{\mathscr{R}} x_{n}\right\|+\delta_{n}\right)^{2}\right]^{1 / 2}
\end{gathered}
$$

for all $n \geq 0$. Indeed, the existence of $x_{0}$ and $y_{0}$ follows from Theorem 7.1. If $x_{n}$ and $y_{n}$ have already been chosen, let $g_{n} \in L^{1}$ 
be such that $\left[g_{n}\right]=[f]-\left[x_{n} \cdot y_{n}\right]$ and $\left\|g_{n}\right\|_{1}<\delta_{n}^{2}$. Then an application of Proposition 8.5 with $\varepsilon, x, y, g$ of that proposition replaced by $\delta_{n+1}, x_{n}, y_{n}, g_{n}$, respectively, yields vectors $x_{n+1}$ and $y_{n+1}$ with the desired properties. The vectors $x$ and $y$ that verify the conclusion of our theorem will be obtained as certain appropriate limits of $\left\{x_{n}\right\}$ and $\left\{y_{n}\right\}$. First let us clarify the choice of $\delta_{n}$. Fix a strictly increasing sequence $\varepsilon_{n} \in(0, \varepsilon)$, and choose $\delta_{n}$ such that

$$
3 \sum_{n=0}^{\infty} \delta_{n}<\varepsilon\|f\|_{1}^{1 / 2}
$$

and

$$
\left(1+\delta_{n+1}\right)\left[\left(\alpha+\delta_{n}\right)^{2}+\left(\beta+\delta_{n}\right)^{2}\right]^{1 / 2} \leq\left(1+\varepsilon_{n+1}\right)\|f\|_{1}^{1 / 2}
$$

whenever $\alpha^{2}+\beta^{2} \leq\left(1+\varepsilon_{n}\right)\|f\|_{1}^{1 / 2}$ and $\alpha, \beta \geq 0$. With this choice we have $\left\|x_{n}\right\| \leq\left(1+\varepsilon_{n}\right)\|f\|_{1}^{1 / 2}$, and hence there exist $n_{1}<n_{2}<$ ... such that $\left\{x_{n_{j}}\right\}_{j}$ converges weakly to a vector $x$ with norm $\|x\| \leq(1+\varepsilon)\|f\|_{1}^{1 / 2}$. The sequence $\left\{y_{n}\right\}$ is Cauchy in norm, and its limit $y$ has norm

$$
\begin{aligned}
\|y\| & \leq\left\|y_{0}\right\|+\sum_{n=0}^{\infty}\left\|y_{n+1}-y_{n}\right\| \\
& \leq\|f\|_{1}^{1 / 2}+\sum_{n=0}^{\infty} 3 \delta_{n} \\
& \leq(1+\varepsilon)\|f\|_{1}^{1 / 2} .
\end{aligned}
$$

To conclude the proof we need only to show that $[x \cdot y]=[f]$ or, equivalently, that $\left\langle T^{k} x, y\right\rangle=\hat{f}(-k)$ for $k \geq 0$. This follows because

$$
\begin{aligned}
\hat{f}(-k) & =\lim _{j \rightarrow \infty}\left[x_{n_{j}} \cdot y_{n_{j}}\right]^{\wedge}(-k) \\
& =\lim _{j \rightarrow \infty}\left\langle T^{k} x_{n_{j}}, y_{n_{j}}\right\rangle \\
& =\left\langle T^{k} x, y\right\rangle . \quad \text { Q.E.D. }
\end{aligned}
$$

\section{REFERENCES}

1. C. Apostol, Ultraweakly closed operator algebras, J. Operator Theory 2 (1979), 49-61.

2. H. Bercovici, A contribution to the theory of operators in the class $A, \mathrm{~J}$. Funct. Anal. 78 (1988), 197-207.

3. __ Factorization theorems for integrable functions, Analysis at Urbana, II, (E. R. Berkson, et al., eds.), Cambridge University Press, 1988. 
4. __ Factorization theorems and the structure of operators on Hilbert space, Ann. of Math. (2) 128 (1988), 399-413.

5. H. Bercovici, C. Foias and C. Pearcy, Dual algebras with applications to invariant subspaces and dilation theory, CBMS Regional Conf. Ser. in Math., No. 56, Amer. Math. Soc., Providence, R.I., 1985.

6. L. Brown, A. Shields and K. Zeller, On absolutely convergent exponential sums, Trans. Amer. Math. Soc. 96 (1960), 162-183.

7. S. Brown, Some invariant subspaces for subnormal operators, Integral Equations Operator Theory 1 (1978), 310-333.

8. _ Contractions with spectral boundary, Integral Equations Operator Theory 11 (1988), 49-63.

9. S. Brown, B. Chevreau and C. Pearcy, Contractions with rich spectrum have invariant subspaces, J. Operator Theory 1 (1979), 123-136.

10. _ On the structure of contraction operators. II, J. Funct. Anal. 76 (1988), 30-55.

11. B. Chevreau, Sur les contractions à calcul fonctionnel isométrique. II, J. Operator Theory (to appear).

12. B. Chevreau and C. Pearcy, On the structure of contraction operators. I, J. Funct. Anal. 76 (1988), 1-29.

13. R. G. Douglas, Banach algebra techniques in operator theory, Academic Press, New York, 1972.

14. K. Hoffman, Banach spaces of analytic functions, Prentice Hall, Englewood Cliffs, 1962.

15. L. Rubel and A. Shields, The space of bounded analytic functions on a region, Ann. Inst. Fourier (Grenoble) 16 (1966), 235-277.

16. B. Sz.-Nagy and C. Foiaş, Harmonic analysis of operators on Hilbert space, North-Holland, Amsterdam, 1970.

17. J. Wermer, On invariant subspaces of normal operators, Proc. Amer. Math. Soc. 3 (1952), 270-277.

Department of Mathematics, Indiana University, Bloomington, IndiANA 47405 